\title{
Prototypes and Particulars: Geometric and Experience-Dependent Spatial Categories
}

\author{
John P. Spencer and Alycia M. Hund \\ University of Iowa
}

\begin{abstract}
People use geometric cues to form spatial categories. This study investigated whether people also use the spatial distribution of exemplars. Adults pointed to remembered locations on a tabletop. In Experiment 1, a target was placed in each geometric category, and the location of targets was varied. Adults' responses were biased away from a midline category boundary toward geometric prototypes located at the centers of left and right categories. Experiment 2 showed that prototype effects were not influenced by cross-category interactions. In Experiment 3, subsets of targets were positioned at different locations within each category. When prototype effects were removed, there was a bias toward the center of the exemplar distribution, suggesting that common categorization processes operate across spatial and object domains.
\end{abstract}

Categorization is a ubiquitous phenomenon seen across a variety of domains. This basic human ability affects how people judge one another (Rothbart, Davis-Stitt, \& Hill, 1997), how they remember the properties of objects and events (Huttenlocher, Hedges, \& Prohaska, 1988; Rosch, Simpson, \& Miller, 1976), and even how they organize actions in local space (e.g., in a fire, grab the items on the desk and race for the door; Barsalou, 1983). But does this pervasiveness reflect the ubiquity of the problem confronting people in various domains or the ubiquity of categorization processes themselves? That is, people may group available information in any domain to make sense of a complex world but use domain-specific processes to form categories. Such an approach to category formation could take advantage of unique cues in each domain. Alternatively, categorization may be a general phenomenon, because domain-general processes operate comparably across domains. In this case, even though the information people use across situations and domains may vary, the same category processes operate on these cues.

John P. Spencer and Alycia M. Hund, Department of Psychology, University of Iowa.

This research was supported by National Institutes of Health Grants RO1 HD22830 and NIMH KO5 MH01102 awarded to Esther Thelen, a research fellowship from Indiana University awarded to John P. Spencer, and a Central Investment Fund for Research Enhancement grant from the University of Iowa awarded to John P. Spencer.

We would like to thank the adults who participated in this study. Gabriel Casciato, Alissa Roste, Anne Schutte, Regan Vinton, and Keith Wilken helped with data collection and analysis. Lloyd Frei, Dexter Gormley, Keith Miller, Dale Parker, and Keiichi Tajima provided valuable assistance in constructing and programming the experimental apparatus. Larissa Samuelson and Gabe Radvansky provided helpful comments on an earlier version of this article. Special thanks go to Esther Thelen. Experiments 1 and 2 would not have been possible without her substantial intellectual and financial support.

Correspondence concerning this article should be addressed to John P. Spencer, Department of Psychology, 11 Seashore Hall E, University of Iowa, Iowa City, Iowa 52242. E-mail: john-spencer@uiowa.edu
To answer this question, one must first identify dissociable "domains" suitable for study. Recent behavioral and neurophysiological data provide support for two dissociable domains or systems central to the present study: the what and where systems (Ungerleider \& Mishkin, 1982). The what system-isolated to cortical areas in a ventral pathway-processes object properties such as form and color. The where system-isolated to cortical areas in a dorsal pathway-processes spatial information such as an object's location. These two systems have been behaviorally and neurally dissociated in numerous studies using nonhuman primates (e.g., di Pellegrino \& Wise, 1993; Miller \& Desimone, 1994; Miller, Erickson, \& Desimone, 1996; Miller, Li, \& Desimone, 1991; Rao, Rainer, \& Miller, 1997; Suzuki, Miller, \& Desimone, 1997; Wilson, Scalaidhe, \& Goldman-Rakic, 1993). In addition, neuropsychological studies with human participants have revealed a dissociation between what and where information (e.g., Levine, Warach, \& Farah, 1985; Shoqierat \& Mayes, 1991). Humphreys and Riddoch (1987), for example, described a patient with agnosia who was able to locate objects but could not recognize them.

The existence of two dissociable domains provides the necessary foundation for testing whether general categorization processes underlie the ubiquity of categorization behaviors across domains. If one can demonstrate that similar categorization processes are at work when people categorize objects and locations, this would suggest that there are general processes that govern the formation of groups independent of the domain in question. Alternatively, if one can demonstrate that different categorization processes are at work when people categorize objects versus when they categorize locations, this would suggest that in some situations unique categorization processes operate within each domain.

This general goal has been pursued across a series of studies by Huttenlocher and her colleagues (e.g., Engebretson \& Huttenlocher, 1996; Huttenlocher, Newcombe, \& Sandberg, 1994; Newcombe, Huttenlocher, Sandberg, Lie, \& Johnson, 1999). These researchers initially applied concepts from the object categorization domain to spatial categorization. Specifically, they proposed a category adjustment (CA) model in which memory responses are 
biased toward geometric "prototypes": the centers of spatial regions (Huttenlocher, Hedges, \& Duncan, 1991). More recently, Huttenlocher, Hedges, and Vevea (2000) applied the CA model to object categorization, introducing a new characteristic of the model: induced categories. According to this new idea, responses should be biased toward the center of induced categories formed by representing the frequency distribution of exemplars within a category.

The present study continued this line of research by examining whether the induced category construct applies to spatial categorization, that is, whether the distribution of target locations within a spatial category affects memory responses. Furthermore, we examined the extent to which induced category effects are separable from geometric prototype effects in the spatial domain. Our data demonstrate that there are, in fact, separable prototype and induced category effects when people categorize locations, suggesting that similar processes underlie categorization in the object and spatial domains. Nevertheless, our data also indicate that some aspects of these processes may be domain specific.

\section{Spatial Categories and the CA Model}

Huttenlocher and colleagues have proposed that similar categorization processes operate when people make a variety of stimulus judgments, including judgments of object size (Huttenlocher et al., 2000), estimates of location from memory (Engebretson \& Huttenlocher, 1996; Huttenlocher et al., 1991; Newcombe et al., 1999), judgments involving event timing and duration (Huttenlocher et al., 1988), and even estimates of grayness (Huttenlocher et al., 2000). This proposal has been formalized in the CA model, which we introduce in the context of location memory tasks, the focus of the present study.

In a typical location memory task, participants are shown a dot inside a geometric frame (e.g., a rectangle) and are asked to remember the dot's location. The frame is then covered up, and participants are asked to place a dot in the same location in a second, blank frame (Huttenlocher et al., 1991; Laeng, Peters, \& McCabe, 1998; Nelson \& Chaiklin, 1980; Sandberg, Huttenlocher, $\&$ Newcombe, 1996). These tasks consistently reveal that people tend to exaggerate the distance between the "midline" axis of a geometric figure and target locations near this axis. Figure 1A shows a schematic of results from a study in which adults were shown a target angle within a " $\mathrm{V}$ " frame and asked to reproduce this angle after a short delay (Engebretson \& Huttenlocher, 1996). Adults' directional responses were biased in the directions indicated by the arrows in the figure: away from midline and away from the edges of the perceptual frame (for related results, see Sandberg et al., 1996; Schiano \& Tversky, 1992; Tversky \& Schiano, 1989). A second example of this response bias is shown in Figure 1B. In this case, participants were asked to remember the location of a dot presented along the horizontal axis of a rectangle. Once again, participants' responses were biased away from midline and away from the edges of the perceptual frame (Huttenlocher et al., 1994).

Huttenlocher and colleagues have proposed that the errors in Figure 1 reflect biases toward the centers of spatial categories formed when people impose category boundaries within a geometric figure (Engebretson \& Huttenlocher, 1996; Huttenlocher et al., 1991; Sandberg et al., 1996). According to their CA model, cate-
A

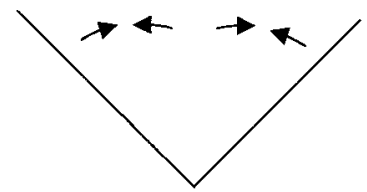

B

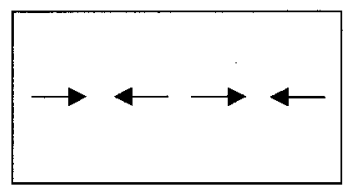

Figure 1. Schematic of biases in remembered location. A: The $\mathrm{V}$ frame task from Engebretson and Huttenlocher (1996). B: The rectangular frame task from Huttenlocher, Newcombe, and Sandberg (1994). Lines with arrows show direction of systematic biases in constant (mean) error. Panel A is from "Bias in Spatial Location Due to Categorization: Comment on Tversky and Schiano," by P. H. Engebretson and J. Huttenlocher, 1996, Journal of Experimental Psychology: General, 125, p. 97. Copyright 1996 by the American Psychological Association. Adapted with permission of the author. Panel B is from "The Coding of Spatial Location in Young Children," by J. Huttenlocher, N. Newcombe, and E. H. Sandberg, 1994, Cognitive Psychology, 27, p. 126. Copyright 1994 by Academic Press. Adapted with permission.

gories help people maintain a memory of a target location because they allow encoding at two levels of detail (Huttenlocher et al., 1991). First, people represent the fine-grained location of the target, for instance, the angular direction of a target within the $\mathrm{V}$ frame. Second, people represent the region or category in which the target was located. More specifically, people represent the central, or prototypical, value within a category, for instance, the direction that runs through the center of the "left" region within the $\mathrm{V}$ frame. The central value is the prototype because it is the most representative member of a category. When asked to reproduce a target direction, participants combine their fine-grained and categorical representations. This leads to systematic biases toward the prototypical values, because all estimates within a region are weighted by the same prototype (Engebretson \& Huttenlocher, 1996). Importantly, the weighting of fine-grained and categorical information can vary in different situations. For instance, people weight prototypical information more heavily when fine-grained information is uncertain, such as when multiple locations must be remembered. Engebretson and Huttenlocher (1996) found larger response biases toward the centers of the left and right regions in the $\mathrm{V}$ task when participants were asked to remember two target directions presented sequentially than when a single target had to be remembered (see also Huttenlocher et al., 1991; Newcombe et al., 1999).

\section{Do Similar Processes Underlie "Prototype" Effects in Location and Object Categorization?}

There are important conceptual similarities between the prototype construct used in the CA model and initial proposals about prototypes in object categorization (e.g., Posner \& Keele, 1968). First, in both domains, the prototype reflects the best representative of the category in question. For instance, in the object domain, prototypes are the most typical category members (e.g., robins are prototypical birds; see Rosch, 1973; Rosch et al., 1976). Similarly, in the spatial domain, the center of a spatial region is the best representative in that it is geometrically farthest from all category boundaries. Second, the prototypical member of a category does 
not have to be experienced as an exemplar to achieve status as a prototype. Original work by Posner and Keele (1968) revealed faster reaction times and lower error rates for novel, prototypical stimuli than for novel, nonprototypical exemplars (for related results, see Rosch et al., 1976). Similarly, prototype effects in the spatial domain appear to emerge even when targets are not presented at the centers of spatial categories. In recent studies involving 2- to 11-year-olds, Spencer and colleagues (Hund \& Spencer, 2001; Schutte \& Spencer, in press; Spencer, Smith, \& Thelen, 2001) found biases toward the center of a spatial region even when no targets were presented at this location.

These similarities across domains suggest that common categorization processes might underlie the formation of categories of objects and locations. Recent data have revealed, however, one potential difference across domains: There may be differences in the origin of prototype effects. In spatial tasks, prototypical locations appear to be primarily determined by the geometry of the task space; prototypes are located at the centers of spatial regions (e.g., Engebretson \& Huttenlocher, 1996; Huttenlocher et al., 1991). By contrast, prototype-like biases in object categorization depend on task-specific experience, that is, on the distribution of exemplars (e.g., Huttenlocher et al., 2000; Koehler, 1996; Kruschke, 1996; Nosofsky, 1986).

\section{Geometric Category Biases in Spatial Tasks}

As discussed previously, many studies investigating category biases in location memory tasks have reported biases toward the centers of spatial regions defined by the geometry of the task space (e.g., Huttenlocher et al., 1994; Laeng et al., 1998; Nelson \& Chaiklin, 1980; Schiano \& Tversky, 1992; Tversky \& Schiano, 1989). Evidence for experience-dependent effects is less conclusive. Originally, Huttenlocher and colleagues (1991) suggested that prototype effects might depend on the distribution of exemplars in the task space. Indeed, they reported subtle changes in the magnitude of bias toward a prototypical distance in a circle-dot task when they modified the distribution of target locations within the circle. These effects were small, however, and were not investigated in great detail. Thus, the general conclusion from studies involving simple location memory tasks is that responses are biased toward the centers of geometrically defined spatial regions.

Nevertheless, studies of spatial priming effects suggest that adults' spatial judgments might be affected by the distribution of targets in the task space (e.g., Clayton \& Habibi, 1991; Curiel \& Radvansky, 1998; McNamara, 1986; McNamara, Halpin, \& Hardy, 1992). For example, McNamara and colleagues (1992) used a spatial priming task to examine whether the spatial and temporal distribution of targets influences spatial categorization. Adults learned the locations of 30 objects in a small rectangle presented on a computer monitor. Items were presented in a predefined order during learning, resulting in four types of critical pairs: items that were spatially close and temporally close, items that were spatially close and temporally far, items that were spatially far and temporally close, and items that were spatially far and temporally far. After learning, participants completed a primed recognition task. Results revealed significant priming effects (i.e., faster verification time) only for pairs of objects that were both spatially and temporally close during learning. Thus, when people learn the locations of nearby objects close together in time, they organize these object locations into groups. Although these findings are consistent with the idea that spatial memory is affected by the distribution of locations within a category, it is not clear from these studies whether such distributional effects arise from spatial categorization processes, object categorization processes, or the mapping of "what" information onto "where" information (for a detailed discussion of these issues, see Curiel \& Radvansky, 1998).

To summarize, results from a variety of studies demonstrate that location memory responses are biased toward the centers of geometrically defined spatial regions. To date, however, no studies have demonstrated that adults' location estimates are affected by both geometric cues and the distribution of targets in the task space.

\section{Experience-Dependent Biases in Object Categorization}

In contrast to spatial categorization, the distribution of exemplars affects object categorization (e.g., Kruschke, 1996; Nosofsky, 1986). One example of such effects central to the present investigation comes from a recent article by Huttenlocher and colleagues (2000) in which they applied the CA model to object categorization. In this study, adults viewed objects that varied along a single perceptual dimension. For instance, in one experiment, participants saw pictures of fish that varied in size ("thin" fish vs. "fat" fish). Each exemplar was shown for several seconds, there was a brief delay, and then participants were asked to adjust the size of a response fish to match the remembered exemplar. Across conditions, Huttenlocher et al. varied two aspects of the within-category exemplar distribution: the specific exemplars to which participants were exposed and the relative frequency of exemplars.

In one experiment, participants were exposed to a uniform, narrow distribution of exemplars, but the specific exemplars within the category varied across conditions. For instance, some participants saw a set of thin fish, whereas other participants saw a set of fat fish (Figure 2A). Participants in these conditions showed biases toward the center stimulus value to which they had been exposed. In the thin fish condition, participants overestimated the size of very thin fish and underestimated the size of midsized fish (Figure 2B). By contrast, participants in the fat fish condition overestimated the size of midsized fish and underestimated the size of very fat fish. In another experiment, Huttenlocher et al. (2000) varied the relative frequency of within-category exemplars. Participants in both conditions were exposed to the same range of stimuli (i.e., thin and fat fish), but fish were presented with equal frequency in one condition and according to a normal distribution in another (Figure 2C). The central results are shown in schematic form in Figure 2D: There was less bias near the edges of the exemplar range in the normal distribution condition than in the uniform distribution condition.

Huttenlocher and colleagues (2000) proposed that the two sets of results depicted in Figure 2 reflect participants' use of induced category information. Induced categories are formed from a representation of observed sets of stimuli. Such categories have a "graded" structure with instances that vary from good (near the central value of the distribution of exemplars) to poor (near the boundaries of the category; see also Kay \& McDaniel, 1978; Rosch, 1975). To formalize how induced categories produce biased estimation, Huttenlocher et al. proposed that people encode 
A

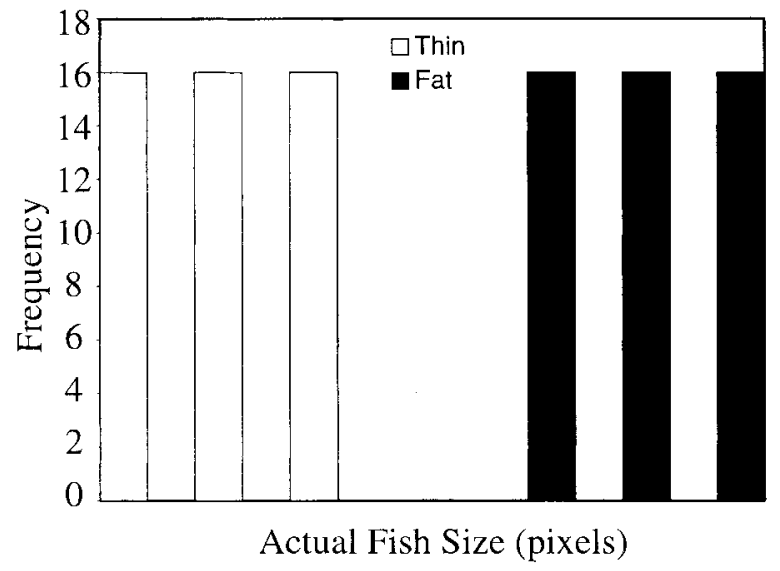

$\mathrm{C}$

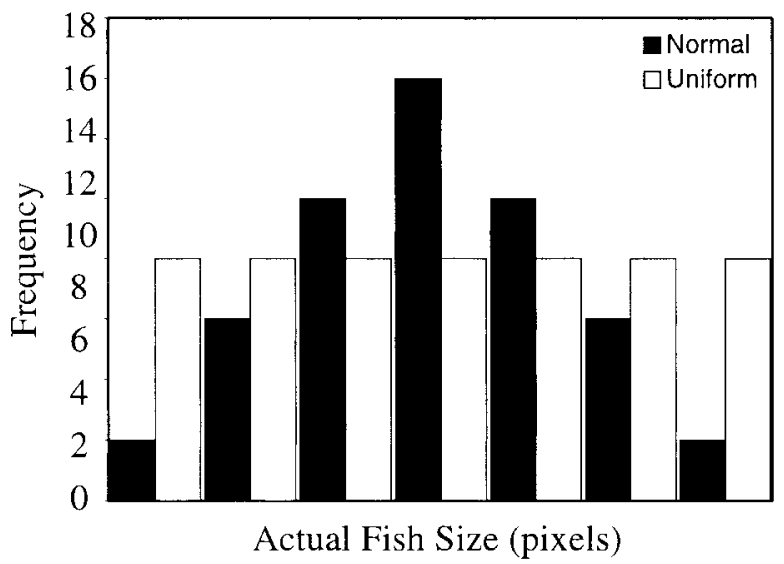

B

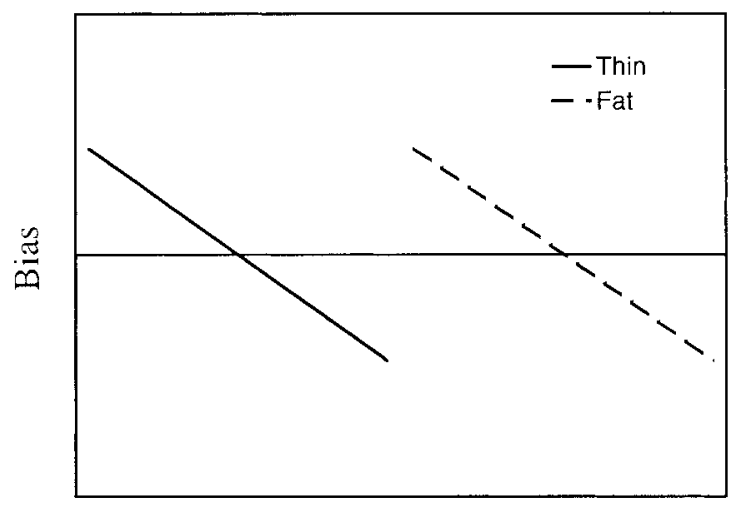

Actual Fish Size

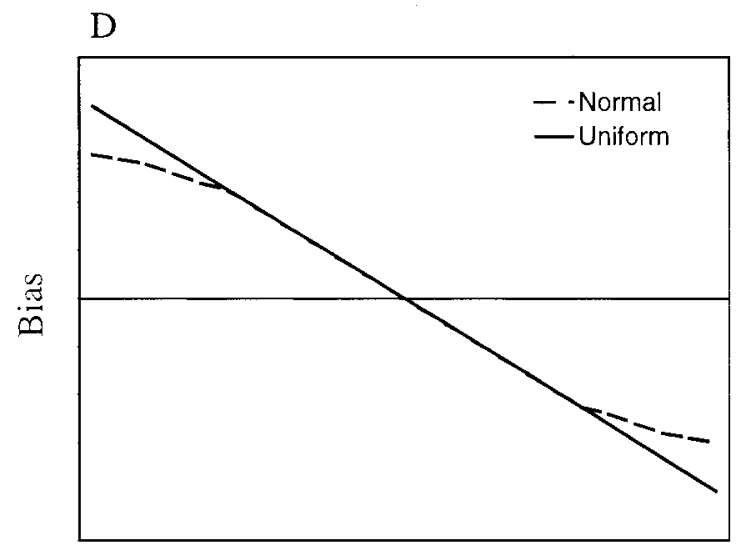

Actual Fish Size

Figure 2. Schematic frequency distributions of stimuli presented by Huttenlocher, Hedges, and Vevea (2000) in the narrow uniform conditions (i.e., the thin fish [white bars] and fat fish [black bars] conditions; Panel A) and the uniform (white bars) and normal (black bars) conditions (Panel C) and schematic of mean response bias in the narrow uniform conditions (i.e., the thin fish [solid line] and fat fish [dashed line] conditions; Panel B) and the uniform (solid line) and normal (dashed line) conditions (Panel D). Positive values reflect overestimation of fish size relative to actual size.

objects at a fine-grained level, such as the exact size of a fish, and at a categorical level, such as the induced category to which a stimulus belongs. At the time of stimulus estimation, people combine these two sources of information. This produces a bias toward the center of the induced category, because all stimulus values within a category are weighted with the same mean. Huttenlocher and colleagues formalized this proposal in a modified version of the CA model.

\section{Is Space Special?}

Inclusion of induced category effects in the CA model raises a fundamental question: Is this model intended to capture a single domain-general categorization process, or does it capture two processes, each of which is specific to a particular domain? For example, this model is domain general because it captures prototype-like effects prevalent in both the spatial and object categorization domains (geometric prototypes in the former case and induced categories in the latter). Furthermore, the geometric prototype and induced category constructs are conceptually similar across domains; both constructs reflect the most representative member of a category. Indeed, both constructs have been mathematically formalized in the same way (see Huttenlocher et al., 1991, 2000). Nevertheless, the CA model appears to be domain specific in that the origin of geometric prototype and induced category effects differs in location and object categorization. In the spatial domain, category effects arise from the geometry of the task space, whereas, in the object domain, category effects depend on the distribution of exemplars. The goal of the present study was to determine whether this domain-specific difference represents a clear divide between location and object categorization.

\section{Specific Goals}

There is clear evidence that estimates of location are biased toward the centers of spatial categories. The central question we 
investigated here is whether the distribution of targets within a spatial category also affects estimates of location. It was not possible to examine this issue in previous studies by Huttenlocher and colleagues because the distribution of targets within spatial categories was uniform (e.g., Engebretson \& Huttenlocher, 1996; Huttenlocher et al., 1991). Thus, the center of the exemplar distribution always overlapped the center of each spatial region.

One way to determine whether responses are biased toward the center of the exemplar distribution is to systematically vary the target locations within a category. Consider the hypothetical example shown in Figure 3A. This example shows targets that vary along one spatial dimension: target direction (see Engebretson \& Huttenlocher, 1996). Targets are positioned on either side of a midline axis within two spatial categories, a "left" category and a "right" category. (Note that, to simplify our discussion, we have distributed targets uniformly within the upper half of the task space, that is, from $-90^{\circ}$ to $90^{\circ}$.) Figure $3 \mathrm{~B}$ shows the frequency distribution typically used in the studies of Huttenlocher and colleagues; participants make one response to each target. With this design, participants would show biases toward the centers of the left and right categories, that is, toward $\pm 90^{\circ}$ (see arrows in Figure 3A). To examine induced category effects, one could expose two groups of participants to a narrow range of targets all contained within one category, such as the "near" and "far" targets in the right category of Figure 3C (for the associated frequency distributions, see Figure 3D). Then, one could measure participants' responses and look for biases toward the center of each distribution: toward $20^{\circ}$ for the near induced category and toward $60^{\circ}$ for the far induced category.

The picture in Figure $3 \mathrm{C}$ is complicated, however, by the presence of spatial prototype effects. Specifically, we would expect to see significant differences across conditions in this hypo-
A

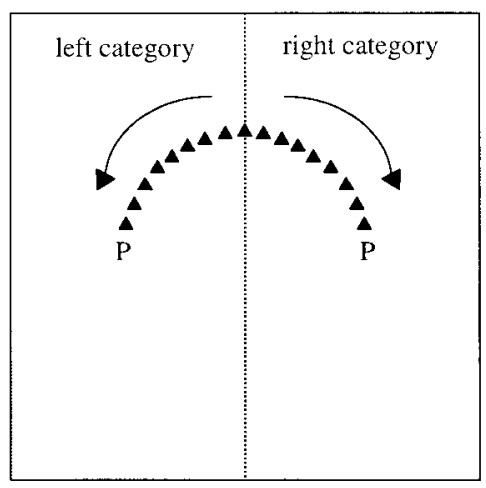

$\mathrm{C}$

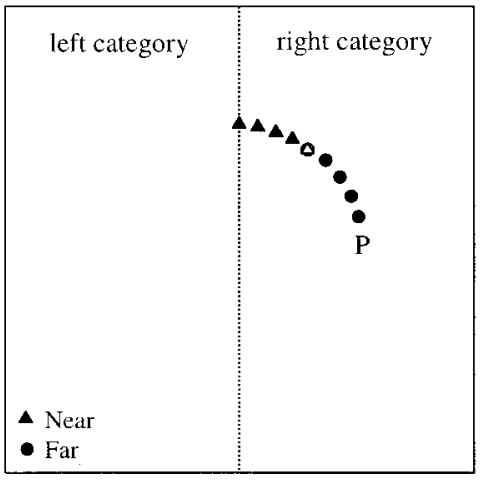

B

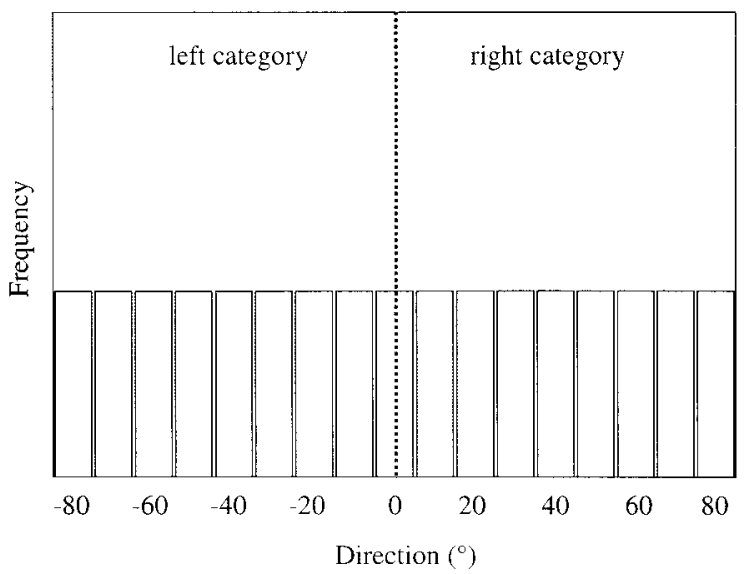

D

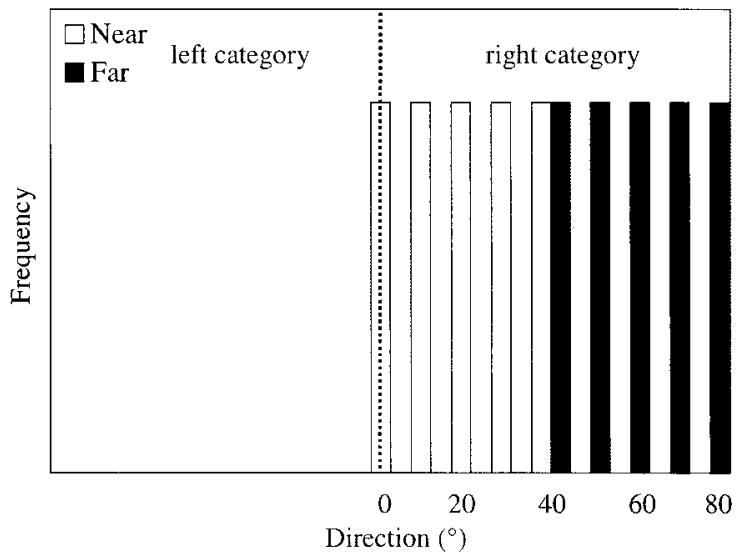

Figure 3. A and B: Target locations and frequency distribution of target presentation from a hypothetical experiment similar to that of Engebretson and Huttenlocher (1996). The dotted lines illustrate the midline axis, which divides the task space into two categories (left and right). Geometric prototypes are marked by Ps. Lines with arrows in Panel A show the direction of systematic biases toward geometric prototypes at $\pm 90^{\circ}$. C and D: Target locations and associated frequency distribution for a hypothetical experiment investigating induced category effects. Triangles in Panel C represent targets in a "near" category; circles represent targets in a "far" category. White bars in Panel D depict target frequencies in the near condition; black bars depict target frequencies in the far condition. 
thetical experiment solely as a result of geometric prototypes. On the basis of previous studies by Huttenlocher and colleagues (see, for example, Engebretson \& Huttenlocher, 1996), participants in this example should make greater outward errors at $20^{\circ}$ than at $40^{\circ}$, because $20^{\circ}$ is farther from the center of the right category (i.e., $90^{\circ}$ ). Similarly, participants should make greater outward errors at $40^{\circ}$ than at $60^{\circ}$, and they should make greater outward errors at $60^{\circ}$ than at $80^{\circ}$. As a consequence, there should be larger response biases to the near category than to the far category, leading to significant condition effects.

In the present study, we used the following strategy to handle this complication. In Experiment 1, we obtained a measure of geometric prototype effects at individual locations in a category (e.g., the right category) by placing one item in the category and measuring the bias toward the prototype. Specifically, across conditions, we varied the placement of single targets as illustrated in Figure 4A and Figure 4B. In Experiment 2, we verified that this strategy provided a valid measure of biases toward geometric prototypes at each location by testing whether items positioned in one category influenced items positioned in a second category (e.g., the left category). According to the CA model (Huttenlocher et al., 1991), items on either side of a certain midline boundary should not interact. Finally, in Experiment 3, we recorded participants' responses as they estimated target locations in a near or far category that were composed of the same absolute spatial locations used in Experiment 1 (see Figure 4C and Figure 4D). The central question was whether participants showed biases toward the centers of the near and far induced categories after geometric prototype effects had been removed.

\section{Experiment 1}

The central goal of this experiment was to measure geometric prototype effects at individual locations in a task space. As discussed earlier, the general strategy was to place one item in each spatial category and vary the within-category location of the target across conditions. Given that the geometric cues were held constant across conditions, this should provide a measure of geometric
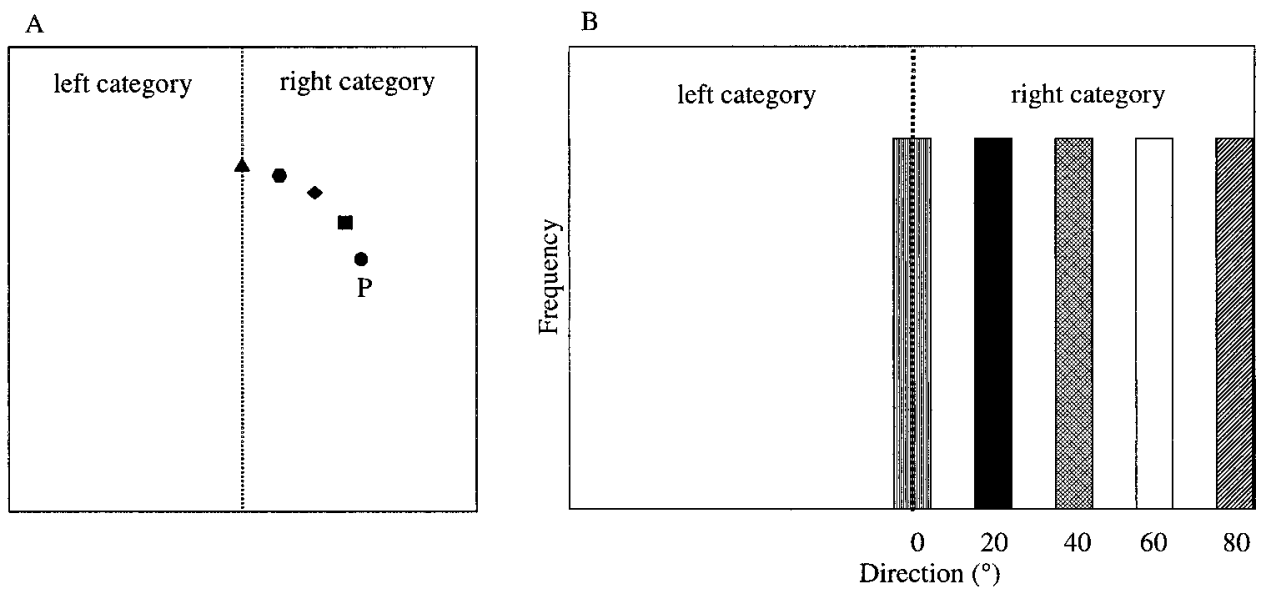

$\mathrm{C}$
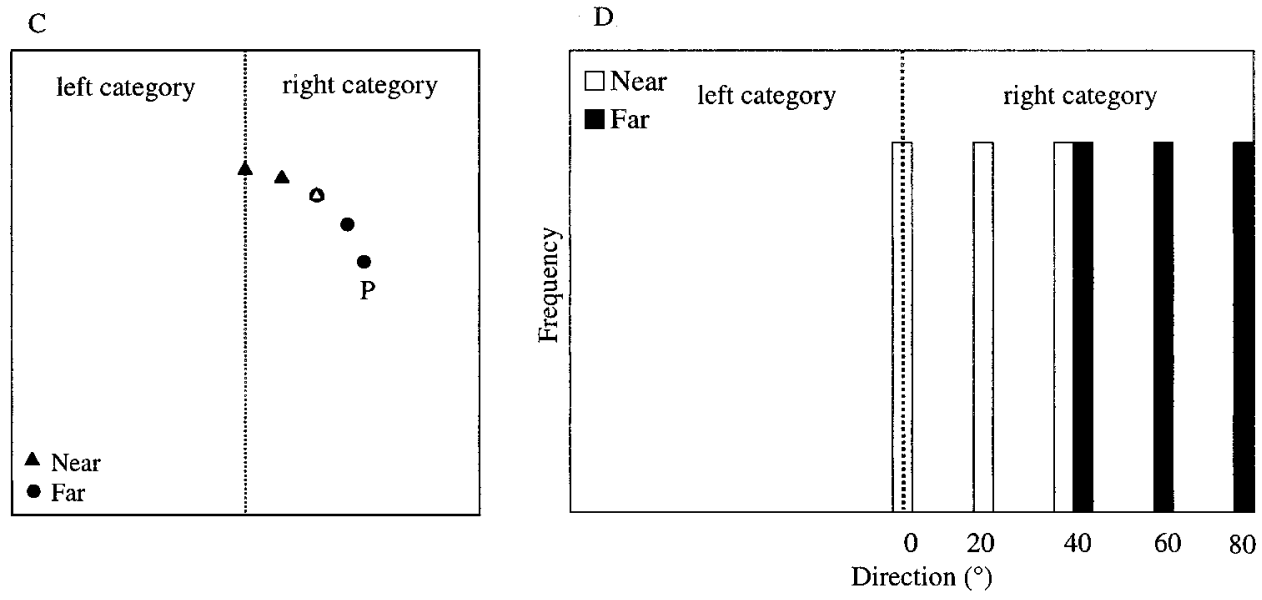

Figure 4. A and B: Target locations and frequency distribution of target presentation within a right category (see Experiment 1). Different symbols in Panel A and associated bar styles in Panel B indicate the single target in each experimental condition. C and D: Target locations and associated frequency distribution for induced category experiment (see Experiment 3). Symbols in Panel C and bar styles in Panel D refer to targets in a "near" and a "far" category, as in Figure 3. The dotted lines illustrate the position of the midline axis. Geometric prototypes are marked by $P$ s. 
prototype effects at each sampled location. Importantly, induced category effects should not be present, because inferences about the exemplar distribution require at least two items within each category (Huttenlocher et al., 2000).

To accomplish this goal, we used a task similar to the paperand-pencil tasks used by Huttenlocher and colleagues (e.g., Engebretson \& Huttenlocher, 1996). Targets were presented on a large, homogeneous tabletop with no explicit visual landmarks (see Figure 5). After a short delay, participants were asked to point to the remembered location. This task has several technical advan-

A Optotrak
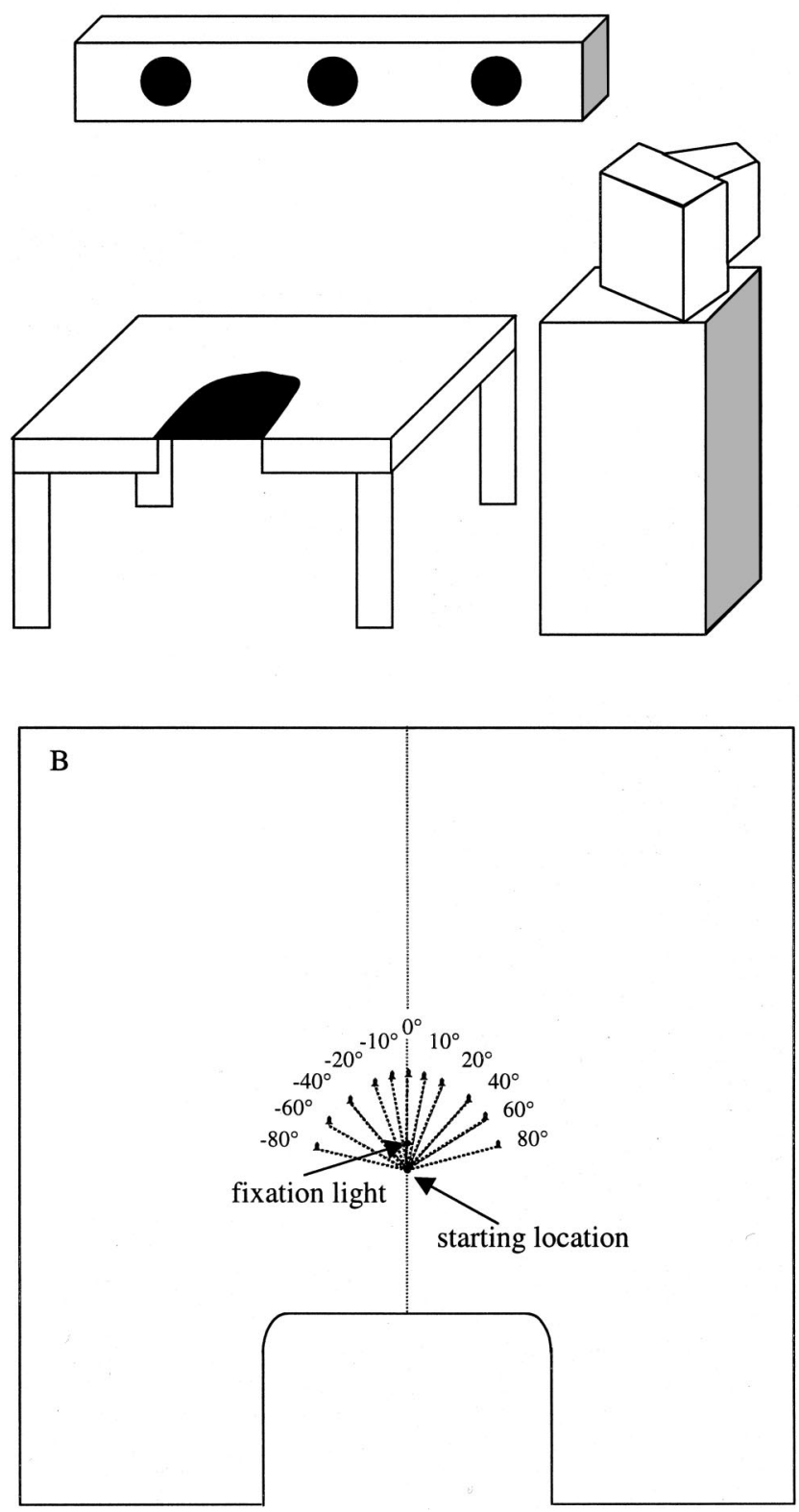

Figure 5. A: Diagram of the experimental table, the Optotrak cameras, and the feedback monitor. B: Overhead view of tabletop with a diagram of the target locations used across experiments. The dashed line shows the midline symmetry axis. tages over paper-and-pencil tasks. First, the timing of events during each trial is precise, because a computer controls target presentation and presentation of a "go" signal after each memory delay. Second, the computer tracks participants' responses with millimeter precision, allowing us to detect even subtle changes in response errors.

A third advantage is that people appear to form relatively simple spatial categories in this task. Specifically, Hund and Spencer (2001) and Spencer and Hund (2001) found that 6- and 11-year-old children used the midline symmetry axis to divide the task space into left and right regions (see dashed line in Figure 5). Given that older children and adults generally categorize space in the same manner (e.g., Sandberg et al., 1996), we expected adults to use the midline axis to form two large spatial categories with geometric prototypes at the centers of each region (i.e., at $\pm 90^{\circ}$ ). This would be consistent with adults' use of midline in other tasks (e.g., Engebretson \& Huttenlocher, 1996; Huttenlocher et al., 1991, 1994). ${ }^{1}$

Rather than having participants estimate the location of only one target across all trials (which could create task demand effects relative to later conditions with multiple items in each category), three targets were included in each condition. One target was in the left category (i.e., to the left of the midline axis of the table), whereas a second target was in the right category. The location of these targets within each category varied across conditions. In addition, we included a third target that was aligned with the midline category boundary. Responses to this target provided an index of the certainty of the category boundary. If the category boundary is relatively certain - as data from Hund and Spencer (2001) and Spencer and Hund (2001) suggest—responses to this target should show zero mean bias and low variability.

A secondary goal of this investigation was to operationalize the certainty construct in the CA model in a way that would not interfere with measurement of induced category effects. According to the CA model, responses should be more strongly biased toward categorical information as fine-grained information becomes less certain (Huttenlocher et al., 1991). As mentioned previously, this proposal has been tested in the spatial domain with interference tasks in which participants must remember two locations. Consistent with the CA model, adults show larger biases toward geometric prototypes in these tasks relative to performance when a single location must be remembered (Engebretson \& Huttenlocher, 1996; Newcombe et al., 1999). Use of an interference task might be

\footnotetext{
${ }^{1}$ Adults might use other symmetry axes as well. They might, for instance, use the diagonal axes of the table. We thought this would be unlikely, however, because diagonal axes are perceived less accurately than vertical and horizontal symmetry axes (e.g., Beh, Wenderoth, \& Purcell, 1971; Cecala \& Garner, 1986; Wenderoth, 1997; Wenderoth, Johnstone, \& van der Zwan, 1989). Moreover, adults sometimes fail to use diagonal axes to categorize space, even when such axes are readily available (Schiano \& Tversky, 1992; Tversky \& Schiano, 1989). Another possible category boundary was the horizontal midline symmetry axis. We expected this axis to be less salient because, when participants were seated within the "arc" cut out from the front of the table (see Figure 5), there was no visible line that extended across the entire front edge of the task space. Axes defined by incomplete reference cues (e.g., the horizontal axis of the table) are less salient that axes defined by visible lines (e.g., the vertical midline axis; see Li \& Westheimer, 1997).
} 
problematic in the present study, however, particularly if the interference item is a member of the same category as the target. According to Huttenlocher and colleagues, interference effects are caused by the interaction between the fine-grained memory of the target item and the interference item. Such an effect may obscure the central interaction we attempted to measure in Experiment 3: interactions between the fine-grained memory of the target item and the memory of all other within-category items (i.e., the induced category).

Thus, rather than using an interference task, we varied the delays during which participants were asked to remember a target location. The rationale was quite simple: As delays increase, fine-grained memory for the target location should become less certain, leading to larger biases toward geometric prototypes. Furthermore, by having participants move repeatedly to each target at each delay, we could obtain converging evidence within participants that geometric prototype effects are linked to the certainty of information in memory. Specifically, constant (mean) error provides an index of prototype-like biases, whereas variable (standard deviation) error provides an index of the stability of memory. If the CA model is correct, we should see an increase in constant error toward geometric prototypes over delays as well as an associated increase in response variability.

Delay-dependent findings in previous studies have been mixed. Tversky and Schiano (Schiano \& Tversky, 1992; Tversky \& Schiano, 1989) failed to find evidence of memory decay across 7 to 10-s delays using a version of the V-frame task shown in Figure 1A. Nevertheless, several recent studies have reported delaydependent effects. Chieffi and Allport (1997) reported a significant increase in both absolute directional and distance error when adults pointed to remembered locations in the dark after delays of 4, 10, and $30 \mathrm{~s}$ (for related results, see Chieffi, Allport, \& Woodin, 1999). Similarly, Elliott and Madalena (1987) found an increase in distance error across delays of 2, 5, and $10 \mathrm{~s}$, and McIntyre, Stratta, and Lacquaniti (1998) found an increase in variable errors over delays of 0.5, 5, and $8 \mathrm{~s}$ (see also McIntyre, Stratta, \& Lacquaniti, 1997). Finally, several recent studies with 3 - to 11-year-old children have shown systematic increases in biases toward geometric prototypes over delays using the same task used here (Hund \& Spencer, 2001; Schutte \& Spencer, in press; Spencer \& Hund, 2001).

\section{Method}

\section{Participants}

Thirty adults $(M$ age $=25$ years 0.43 months, $S D=80.44$ months $)$ participated in this study. Data were collected from 2 additional adults; however, these data were not included in the final analyses because one of the participants was missing data from more than $10 \%$ of all trials and the other participant repeatedly moved to the wrong target location, even on trials with no delay. Undergraduate and graduate students and staff were recruited at Indiana University and the University of Iowa, and introductory psychology students were recruited at the University of Iowa. Fourteen participants (including the 2 not included in the final analyses) completed the experiment at Indiana University, and the remaining 18 participated at the University of Iowa. All participants were right-handed. The number of women and men was roughly balanced across conditions.

\section{Apparatus and Materials}

Participants sat at a $1.22-\mathrm{m} \times 1.22-\mathrm{m}(4-\mathrm{ft} \times 4$-ft) horizontal table, the top of which was a uniform piece of Plexiglas. An arc was removed from one side of the table, and participants were seated in an adjustable chair positioned within this arc with the tabletop at belly height (see Figure 5). In this orientation, the tabletop extended to both sides of the participants, limiting their ability to use the front edge of the table as a reference axis. The Plexiglas tabletop was covered with black tinting on the underside to prevent participants from seeing the small light-emitting diodes (LEDs) positioned below. In addition, the room lights were dimmed, and black cloth was hung across the ceiling and down the walls to prevent reflections from appearing on the tabletop. After these adjustments, the top of the table appeared to be a smooth, black, homogeneous surface.

A small (1-cm-radius) yellow sticker was placed along the midline axis of the table $30 \mathrm{~cm}$ from the front edge. This served to mark the starting location for each trial. An electromagnetic switch positioned just below the starting location (under the Plexiglas tabletop) was used to ensure that participants were ready to begin each trial, remained at the starting location during the delays, and started moving at the correct time.

Targets were illuminated through a bank of LEDs with diodes every $10^{\circ}$ from $-90^{\circ}$ to $90^{\circ}$. The diodes were located $15 \mathrm{~cm}$ from the starting location, and a fixation light diode was placed $4 \mathrm{~cm}$ in front of the starting location. The diodes were embedded within a piece of black Plexiglas. An $\mathrm{X}$-ray film covered the LED board. The film was opaque except for spaceship shapes $(1.25 \mathrm{~cm}$ from the tip to the base, $1.25 \mathrm{~cm}$ across the base, and $0.65 \mathrm{~cm}$ at the midsection) aligned with the LEDs and a circle $(0.5-\mathrm{cm}$ radius) aligned with the fixation light. A thin piece of white paper on top of the X-ray film diffused the light from the LEDs. LED voltage was adjusted to avoid visual afterimages.

The lights and switch were controlled by a personal computer equipped with an input-output board connected to an electromechanical relay box. The relays were able to trigger the LEDs with better than 10-ms precision. The computer controlled the type and timing of all stimuli using customized software. The computer monitor was used to present visual feedback after each trial. The monitor was positioned to the right of the table at a comfortable viewing distance (see Figure 5). The experimenter sat to the right of the experimental table, next to the computer monitor, during data collection sessions. Prerecorded messages were played through two speakers placed on the floor on either side of the table. These messages led participants through the spaceship game and gave them praise or warning messages after each trial.

Participants' movements of the dominant, right finger were recorded with an optical electronic motion analysis system (Optotrak, Northern Digital). Optotrak tracks small (radius: $3.5 \mathrm{~mm}$ ), individually pulsed infrared-emitting diodes (IREDs) within a specified three-dimensional volume with better than $1-\mathrm{mm}$ precision. Pointing data were collected at $150 \mathrm{~Hz}$ in a predefined coordinate system. The $(0,0)$ coordinate was positioned at the starting location. The " $\mathrm{x}$ " coordinate axis was defined as the left-right dimension, and the " $y$ " coordinate axis was defined as the front-back dimension.

\section{Task and Procedure}

When participants arrived at the laboratory, they were told that they were going to play a game to find spaceships. They were seated at the experimental table and told that spaceships would appear and then disappear somewhere on the tabletop in front of them. The participants' task was to remember where each spaceship was and to move to the remembered location at the end of a "ready, set, go" sequence spoken by the computer. Participants moved to the target locations by sliding a magnetic disk along the tabletop. The magnet was covered with felt on one side so that it would glide easily on the Plexiglas. After the game was described, participants signed consent forms. Three IREDs were placed on participants' right index finger. Then participants were seated at the experimental table, and 
a brief practice session began. During practice, the specific task and types of feedback were explained.

Each trial began when the computer stated, "beginning search for enemy spaceships." Next, participants moved the magnet to the starting location and attended to the table in front of them. After a random pretrial delay ranging from 2-4 s, a "spaceship" light was illuminated for $2 \mathrm{~s}$. Participants then heard a "ready, set, go" sequence. This sequence ended $0,5,10$, 15 , or $20 \mathrm{~s}$ after the target disappeared. As a means of controlling participants' looking direction during the 5- to 20-s delays, a fixation light appeared after the target disappeared. Participants were asked to look at this light, rather than looking at the spaceship's location. The experimenter made sure participants looked at the fixation light on each trial. The fixation light was turned off at the start of the "ready, set, go" sequence.

Participants were instructed to move directly to where they thought the spaceship was located when they heard "go." Movement speed was not emphasized; however, initiation time relative to the go signal was emphasized. This ensured that the length of the delay periods remained relatively constant across trials and participants. Participants were also told that they could make small corrections at the end of the movement to find the exact spot where they thought the spaceship was located. They were asked to maintain this final position until they received feedback from the computer. At the end of each 3.5-s trial, the target was reilluminated for $1.5 \mathrm{~s}(2 \mathrm{~s}$ during the practice phase of Session 1). This allowed participants to compare the location of their finger (the remembered target location) with the actual target location. Then feedback information was displayed on the computer monitor for $2 \mathrm{~s}$. After feedback, the screen was blanked, there was a short delay, and the computer began the next trial. We chose to provide feedback to keep participants interested in the task. This also allowed for more direct comparisons with children's performance, a longer term goal of the present research (see Hund \& Spencer, 2001; Schutte \& Spencer, in press; Spencer \& Hund, 2001). Young children (e.g., 6-yearolds) require extensive feedback to maintain their interest in the task.

Computer feedback was based on two sources of information. First, the computer computed movement initiation time relative to the go signal. Initiation time scores ranged from 5 points for responses begun within \pm 40 $\mathrm{ms}$ of the go signal to 0 points for responses begun more than $200 \mathrm{~ms}$ before or after the signal. Second, the computer computed an accuracy score based on the Optotrak data. Accuracy scores ranged from 5 points for responses $0-1 \mathrm{~cm}$ from the target to 0 points for responses $3 \mathrm{~cm}$ or greater from the target.

Four types of feedback information were displayed on the computer screen after each trial: (a) initiation time relative to the go signal, (b) the sum of the accuracy and initiation time points, (c) total accumulated points, and (d) a "flight rank." Point scores of 9 and 10 were accompanied by "good job" and "direct hit" messages, respectively. Participants received a warning message from the computer when their initiation times were at the boundaries of the acceptable range.

\section{Experimental Design}

Participants were randomly assigned to one of five experimental conditions. In each condition, participants moved to three target locations: a left, center, and right location. One target was presented to the left of the midline axis of the table (i.e., in the left category), and one target was presented to the right of midline (i.e., in the right category). The angular distance of the left and right targets from midline varied across separation conditions; targets were $10^{\circ}, 20^{\circ}, 40^{\circ}, 60^{\circ}$, or $80^{\circ}$ from the midline axis (see Figure 5). The center target was always aligned with the midline category boundary $\left(0^{\circ}\right)$, regardless of separation condition. This target provided an index of boundary certainty. The use of three targets kept the total number of targets identical across the experiments reported in this article.

Each participant came into the laboratory for two experimental sessions. During the first session, participants completed 15 practice trials, 5 to each target location at randomly selected delays. After practice, they completed 60 trials in blocks of 15 trials each. There were 4 trials to each target at each of four delays $(5,10,15$, and $20 \mathrm{~s})$. Session 2 was identical to Session 1 except that there were only 6 practice trials at the start of the session. All trials were randomized with the constraint that responses to one target location never occurred more than twice in succession. In addition to these trials, participants completed four 0-s delay trials to each target per session. On these trials, the target remained illuminated after the "ready, set, go" sequence. Thus, these trials did not place memory demands on the participants.

\section{Data Analysis}

Three-dimensional Optotrak data were filtered with a 2nd-order recursive low pass Butterworth filter with a $20 \mathrm{~Hz}$ cutoff. This cutoff frequency was determined through the residual analysis proposed by Winter (1990). After filtering, Optotrak data from each trial were analyzed with customized computer software. First, the computer selected three kinematic events to identify potentially valid $x$ and $y$ coordinates at the end of each response: the end of the "transport" phase, the end of the "correction" phase, and the end of the "extra" correction phase. These kinematic events were selected according to the following rules. The end of the transport phase was defined by one of two possible events: the first tangential velocity minimum or the first data frame less than $2 \mathrm{~cm} / \mathrm{s}$ after the velocity dropped below $30 \%$ of the peak velocity and stayed below $30 \%$ for at least one more velocity peak. The end of the correction phase was defined as the first data frame less than $2 \mathrm{~cm} / \mathrm{s}$ after the transport phase with a peak correction velocity above $4 \mathrm{~cm} / \mathrm{s}$ and less than $30 \%$ of the peak velocity. The $4-\mathrm{cm} / \mathrm{s}$ criterion ensured that the computer would search for a velocity peak after the transport phase with a maximum greater than the resting velocity (2 $\mathrm{cm} / \mathrm{s}$ ). If no valid velocity peak was found, the computer simply selected the first velocity minimum below the resting velocity. The rules for identifying the correction phase were also used to identify the end of the extra correction phase. The most accurate kinematic event was included in the final analyses. This was the most conservative choice because it maximized participants' accuracy, which was counter to the goal of investigating increases in memory errors across delays.

After the computer had selected the most accurate kinematic event on each trial, directional errors at these locations were computed. For each $x-y$-coordinate pair, the angle between the start-end line and the starttarget line was calculated. Given that our primary goal was to measure biases toward geometric prototypes-that is, away from the category boundary-directional errors were computed such that positive directional errors indicated errors away from the midline axis of the table relative to the target direction, whereas negative errors indicated errors toward the midline axis. Thus, for responses to targets on the left side of the table, a clockwise error would produce a negative directional error (i.e., toward midline). Conversely, a clockwise error for a target on the right side of the table would yield a positive directional error (i.e., away from midline). For responses to the center target, which was aligned with midline, positive directional errors indicated counterclockwise errors.

Directional errors were checked for outliers in a three-stage process. In the first stage, the computer identified all trials in which directional errors were larger than the median error \pm 2 standard deviations for responses to each target location at each delay. Values were set to a minimum value of $5^{\circ}$ for very small standard deviations and a maximum value of $10^{\circ}$ for very large standard deviations. These values were selected on the basis of inspection of standard deviation distributions across all participants. Next, outlier trials were reanalyzed through an interactive version of the automated analysis software to determine whether the large errors were due to computer selection mistakes. Third, the criteria from the first stage were used to eliminate all remaining trials with large directional errors. Trials with initiation times above $1 \mathrm{~s}$ were also eliminated. Three trials did not meet the initiation time criterion. Overall, $0.20 \%$ of all trials were eliminated after the outlier analyses. 
Finally, inspection of the data revealed that the IREDs were slightly elevated and rotated away from midline for responses to the left and right targets on all trials, including the 0-s delay trials when the targets were visible. This occurred because the IREDs were placed on top of the index finger, which naturally rotates away from midline when people point to targets to the left and right of this axis. To eliminate this target location difference, which was due solely to the data collection method, we subtracted the mean directional error for the 0 -s delay trials to each target for each participant from the directional errors to the corresponding target at the 5- to 20-s delays. These adjusted directional errors were used in all subsequent analyses. ${ }^{2}$

\section{Results}

The primary goal of this experiment was to examine how biases toward geometric prototypes vary over delays when targets are presented at different locations within the left and right spatial categories. Thus, we analyzed constant directional errors to the left and right targets at each delay to determine the magnitude of bias toward the centers of the left and right regions (i.e., $\pm 90^{\circ}$ ). In addition, we analyzed variable errors for these targets to determine whether memory became less certain over delays, as predicted by the CA model. In a final set of analyses, we examined responses to the center target $\left(0^{\circ}\right)$. Responses to this target provided an index of the certainty of the midline category boundary.

\section{Geometric Prototype Effects: Responses to the Left and Right Targets}

Constant directional error. Figure 6 shows mean constant directional errors across delays for targets located at each angular separation from midline. Given the symmetry of results across the left and right spatial categories, we have averaged errors for identical locations in the left and right categories (e.g., $-10^{\circ}$ and $10^{\circ}$ ). Inspection of Figure 6 reveals that, in general, responses were

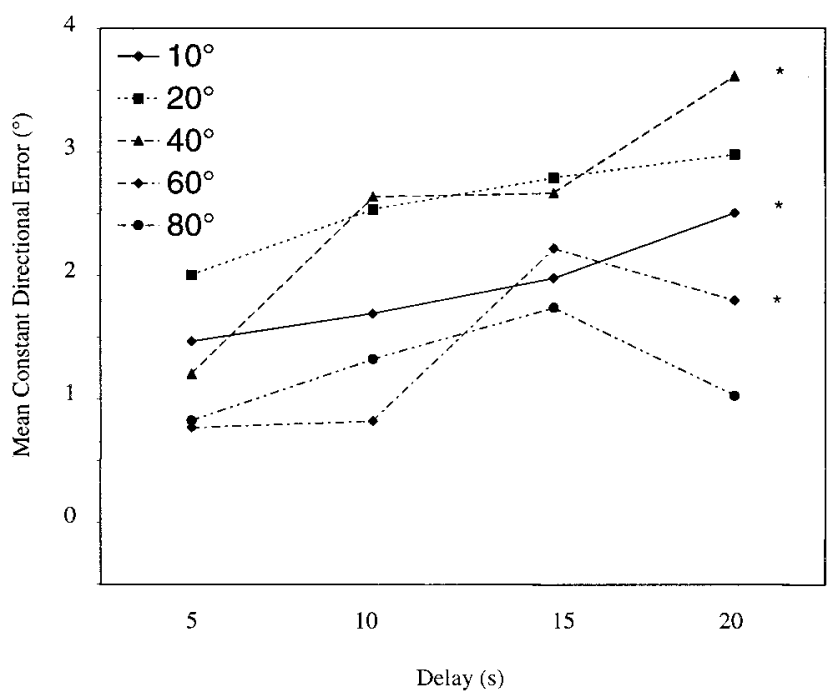

Figure 6. Mean constant directional errors (averaged across responses to targets in the left and right categories) for each separation condition at each delay (Experiment 1). Positive values reflect errors away from the midline of the table, that is, toward spatial prototypes. Asterisks indicate significant delay-dependent effects $(p<.025)$. positive, that is, biased away from the midline category boundary and toward the category prototypes at $\pm 90^{\circ}$. These errors increased systematically over delays, suggesting that bias toward the prototypes increased as uncertainty increased. Moreover, bias was largest at $20^{\circ}$ and $40^{\circ}$ and decreased at the large separations (e.g., $80^{\circ}$ ) as the distance between the target location and the prototypes decreased.

Mean constant directional errors were examined in a three-way analysis of variance (ANOVA) with separation $\left(10^{\circ}, 20^{\circ}, 40^{\circ}, 60^{\circ}\right.$, or $\left.80^{\circ}\right)$ as a between-subjects variable and delay $(5 \mathrm{~s}, 10 \mathrm{~s}, 15 \mathrm{~s}$, or $20 \mathrm{~s}$ ) and side (left or right) as within-subject variables. ${ }^{3}$ Results revealed a significant main effect of delay, Wilks's $\Lambda=.38, F(3$, $23)=12.65, p<.001$, that was subsumed by a significant Delay $\times$ Separation interaction, Wilks's $\Lambda=.43, F(12$, $61)=1.94, p<.05$. Tests of simple effects indicated that constant errors differed significantly across separations at the 5-s delay, $F(4,82)=4.57, p<.025$; the 10 -s delay, $F(4,82)=6.58, p<$ .025 ; and the 20-s delay, $F(4,82)=12.92, p<.025$, but not at the 15-s delay, $F(4,82)=2.35, n^{4}{ }^{4}$ As can be seen in Figure 6, directional biases toward the prototypes at the 5-, 10-, and 20-s delays were generally greatest in the $20^{\circ}$ and $40^{\circ}$ conditions and decreased in magnitude as targets were moved closer (i.e., $10^{\circ}$ ) and further (i.e., $60^{\circ}$ or $80^{\circ}$ ) from the midline category boundary.

Additional simple effects tests indicated that biases toward geometric prototypes increased significantly across delays as uncertainty increased. Specifically, biases increased significantly across delays in three of the separation conditions: $10^{\circ}, F(3$, $75)=3.29, p<.025 ; 40^{\circ}, F(3,75)=3.95, p<.025$; and $60^{\circ}$, $F(3,15)=4.63, p<.025$. Inspection of Figure 6 suggests that delay effects did not reach significance in the $20^{\circ}$ condition, $F(3$, $75)=0.90, n s$, because biases toward the prototype were already quite large at the 5-s delay and plateaued thereafter. Figure 6 also shows that biases toward geometric prototypes were relatively small in the $80^{\circ}$ condition, $F(3,75)=1.22, n s$, probably because the $80^{\circ}$ targets were near the category centers.

In addition to the Delay $\times$ Separation interaction, results of the overall ANOVA revealed a significant main effect of side, Wilks's $\Lambda=.72, F(1,25)=9.52, p<.01$. Although results in the left and right categories were similar, biases toward geometric prototypes (i.e., positive directional errors) were larger in the left category $\left(M=2.52^{\circ}\right)$ than in the right category $\left(M=1.34^{\circ}\right)$. No other results from the overall ANOVA reached statistical significance.

Variable directional error. Next, we examined the variability (standard deviation) of participants' responses to the left and right targets across delays and separations. Because variability provides an index of uncertainty, analyses of variable errors were used to provide converging evidence that delay-dependent increases in bias toward geometric prototypes resulted from increases in un-

\footnotetext{
${ }^{2}$ The analyses of variance described in the Results sections were conducted with both adjusted and nonadjusted data. There were few substantive differences; thus, only analyses of adjusted data are reported.

${ }^{3}$ We used multivariate tests of within-subject variables (Wilks's lambda) in all overall analyses because these tests do not require the assumption of sphericity. Thus, they are more conservative than conventional univariate tests of within-subject variables.

${ }^{4}$ We used a lower alpha level $(p<.025)$ for all follow-up tests to reduce the familywise error rate.
} 
certainty. Figure 7 shows mean variable directional errors averaged across the left and right targets in each separation condition at each delay. As can be seen in this figure, variability generally increased over delays in all separation conditions. Thus, responses did indeed become less certain over delays. In addition, variability was generally smaller at the smallest (i.e., $10^{\circ}$ ) and largest (i.e., $80^{\circ}$ ) separations. These small variable errors may reflect relatively certain encoding of locations close to the midline category boundary (i.e., $0^{\circ}$ ) and close to the prototypes (i.e., $\pm 90^{\circ}$ ).

Mean variable (standard deviation) directional errors were analyzed in a three-way ANOVA with separation as a betweensubjects variable and delay and side as within-subjects variables. Results revealed significant main effects of delay, Wilks's $\Lambda=$ $.60, F(3,23)=5.13, p<.01$, and side, Wilks's $\Lambda=.78, F(1$, $25)=7.22, p<.05$. These main effects were subsumed by a significant Delay $\times$ Side $\times$ Separation interaction, Wilks's $\Lambda=$ $.42, F(12,61)=2.00, p<.05$. Tests of simple effects revealed that variability increased significantly over delays at $20^{\circ}, F(3$, $85)=7.27, p<.01$, and $40^{\circ}, F(3,85)=4.77, p<.01$, but not at the other separations. Although these data are not completely consistent with the constant error results on a target-by-target basis (e.g., there was not a significant increase in constant error over delays at $20^{\circ}$ ), they demonstrate that memory became less certain over delays, as predicted by the CA model.

Additional tests of simple effects indicated that variability was greater on the left side of the table than on the right side, suggesting that memory for locations in the left category is less certain than memory for targets in the right category. Specifically, variability was significantly greater in the left category than the right at $60^{\circ}, F(1,85)=12.96, p<.01$ (left: $M=3.37^{\circ}$; right: $M=2.59^{\circ}$ ), and at $10^{\circ}, F(1,85)=6.32, p<.025$ (left: $M=2.87^{\circ}$; right: $\left.M=2.32^{\circ}\right)$. Similarly, the delay-dependent increase in variability at $20^{\circ}$ was greater in the left category, $F(3$, $75)=10.06, p<.025\left(5 \mathrm{~s}: M=2.93^{\circ} ; 10 \mathrm{~s}: M=2.75^{\circ} ; 15 \mathrm{~s}:\right.$ $\left.M=2.89^{\circ} ; 20 \mathrm{~s}: M=5.00^{\circ}\right)$, than the right, $F(3,75)=0.57, n s$

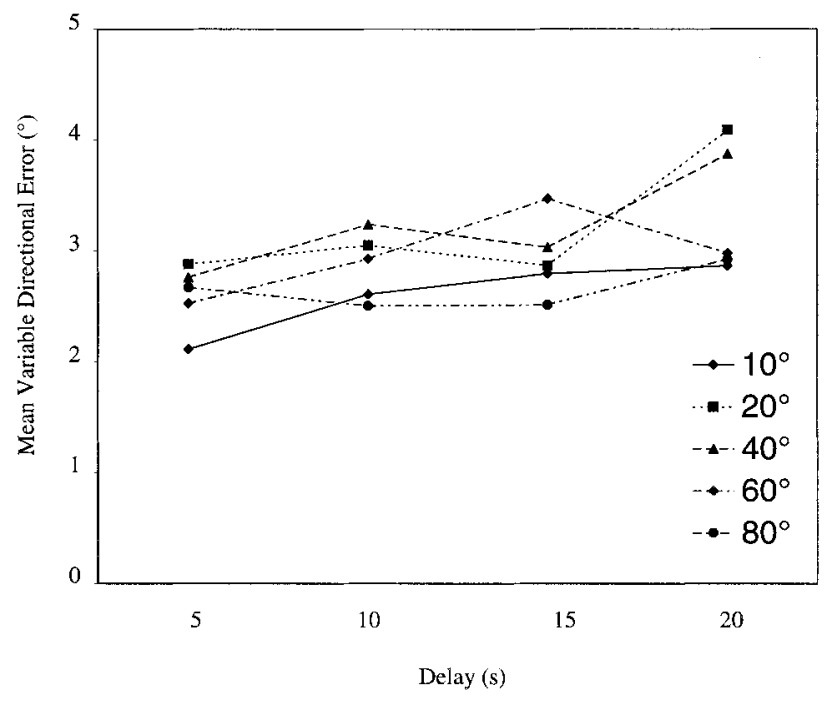

Figure 7. Mean variable (standard deviation) directional errors (averaged across responses to targets in the left and right categories) for each separation condition at each delay (Experiment 1). $\left(5 \mathrm{~s}: M=2.83^{\circ} ; 10 \mathrm{~s}: M=3.35^{\circ} ; 15 \mathrm{~s}: M=2.85^{\circ} ; 20 \mathrm{~s}:\right.$ $M=3.18^{\circ}$ ). The delay-dependent increase in variability at $40^{\circ}$ also differed depending on side; however, the increase at this location was larger in the right category, $F(3,75)=3.84, p<.025(5 \mathrm{~s}$ : $\left.M=2.38^{\circ} ; 10 \mathrm{~s}: M=3.33^{\circ} ; 15 \mathrm{~s}: M=3.71^{\circ} ; 20 \mathrm{~s}: M=3.84^{\circ}\right)$, than in the left, $F(3,75)=3.52, p<.025\left(5 \mathrm{~s}: M=3.14^{\circ} ; 10 \mathrm{~s}:\right.$ $\left.M=3.15^{\circ} ; 15 \mathrm{~s}: M=2.36^{\circ} ; 20 \mathrm{~s}: M=3.92^{\circ}\right)$. Together, these findings generally reveal greater variability in the left category, paralleling the larger prototypical biases in the left category. No other results from the overall ANOVA reached significance.

\section{Certainty of the Category Boundary: Responses to the Center Target}

In a final set of analyses, we examined data from the center target $\left(0^{\circ}\right)$ to assess the certainty of the midline category boundary. If midline is a certain category boundary, then we would expect constant and variable directional errors for the center target to be small in magnitude, because this target was aligned with the category boundary. Figure $8 \mathrm{~A}$ depicts mean constant directional errors for the center target at each delay in each condition, and Figure 8B shows mean variable directional errors across delays and conditions. Inspection of this figure reveals that constant and variable directional errors for the center target were quite small across delays in all conditions, suggesting that midline served as a certain category boundary.

To examine these effects in detail, we entered mean constant directional errors for the center target into a two-way ANOVA with separation $\left(10^{\circ}, 20^{\circ}, 40^{\circ}, 60^{\circ}\right.$, or $\left.80^{\circ}\right)$ as a between-subjects variable and delay ( $5 \mathrm{~s}, 10 \mathrm{~s}, 15 \mathrm{~s}$, or $20 \mathrm{~s})$ as a within-subjects variable. Results revealed no significant effects. Thus, the small positive (counterclockwise) constant directional errors for the center target shown in Figure 8A did not differ significantly across delays or separation conditions. Similarly, mean variable directional errors for the center target were entered into a two-way ANOVA with separation as a between-subjects variable and delay as a within-subject variable. Once again, this analysis revealed no significant results.

\section{Discussion}

There were two main goals of this experiment. The first goal was to measure the magnitude of bias toward the centers of the left and right categories when a single target was placed at different locations within each category. As in our previous studies with children (Hund \& Spencer, 2001; Spencer \& Hund, 2001), targets on either side of the midline axis of the table were biased outward toward geometric prototypes located at $\pm 90^{\circ}$. The magnitude of bias at the left and right target locations varied systematically with target-prototype distance. Constant errors were largest at $20^{\circ}$ and $40^{\circ}$ and decreased systematically as the targets were positioned closer to the prototypes (i.e., at $60^{\circ}$ and $80^{\circ}$ ). This dependency of constant errors on prototype-target distance generally parallels results near midline from other studies (e.g., Engebretson \& Huttenlocher, 1996; Huttenlocher et al., 1994; Sandberg et al., 1996). In addition to these effects, responses to the center target were accurate with small constant and variable errors, suggesting that the midline category boundary was relatively certain. As discussed previously, this category boundary was defined by two visible 

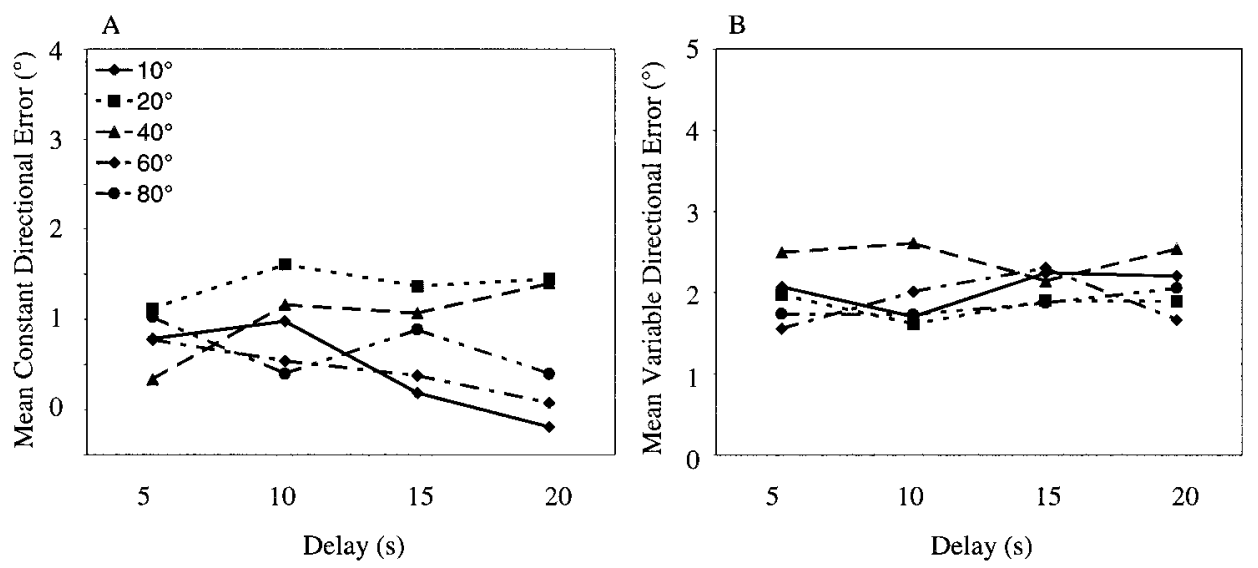

Figure 8. A: Mean constant directional errors for responses to the center target $\left(0^{\circ}\right)$ in each separation condition at each delay (Experiment 1). Positive errors reflect counterclockwise errors. B: Mean variable directional errors for responses to the center target.

reference lines-the edges of the table-making it more perceptually salient than other symmetry axes in the task space. Moreover, we suspect that certainty of midline was enhanced by the presence of the starting location and fixation light, both of which were aligned with this axis.

The second goal of this experiment was to measure changes in bias toward geometric prototypes as the certainty of fine-grained information was manipulated. As predicted by the CA model, participants showed a systematic increase in bias over delays to the left and right targets at all locations except $20^{\circ}$ and $80^{\circ}$. In addition, memory became less certain (i.e., more variable) over delays, particularly to targets far from the prototypes: $20^{\circ}$ and $40^{\circ}$. We suspect that the lack of significant constant error effects at $20^{\circ}$ was caused by the strong categorical bias at this location at delays as short as $5 \mathrm{~s}$. By contrast, the lack of significant constant and variable error effects at $\pm 80^{\circ}$ probably reflects the proximity of these targets to the centers of the left and right categories. The delay-dependent results from the present experiment are consistent with results from several recent location memory studies (Chieffi \& Allport, 1997; Chieffi et al., 1999; Elliott \& Madalena, 1987; McIntyre et al., 1997, 1998).

One final set of results is worthy of note. Participants' memory of target locations in the left category was less certain than their memory of targets in the right category. Similarly, geometric prototype effects were stronger on the left side of the table than the right. It is possible that these effects are related to handedness, because all of our participants were right-handed and responded by pointing with the dominant hand. Data from several studies demonstrate that adults are less skilled at reaching to targets on the nondominant side of the task space (Carnahan, 1998; Fisk \& Goodale, 1985). However, recent data from Haggard, Newman, Blundell, and Andrew (2000) show that, in some cases, adults' perception of location is actually better on the nondominant side of the task space. Moreover, unpublished data from our laboratory indicate that right-handed and left-handed children showed similar biases toward geometric prototypes. Despite a lack of clarity in the origin of these left-right differences, they demonstrate that constant and variable errors covary in the manner predicted by the CA model: As memory becomes less certain, categorical biases increase.

In summary, data from the present experiment are consistent with the predictions of the CA model. As memory became less certain over delays, responses to the left and right targets showed a stronger categorical bias, and the magnitude of this bias varied systematically with target-prototype distance. Thus, these data provide a measure of bias toward geometric prototypes at individual locations in the left and right categories, the foundation needed for investigating induced category effects.

\section{Experiment 2}

Before examining whether spatial memory is affected by induced category information, we conducted a second experiment to investigate the validity of the measure of geometric prototype effects obtained in Experiment 1. In particular, the present experiment investigated whether this measure was "contaminated" by cross-category interactions.

According to the CA model, when two targets in adjacent categories (e.g., left and right categories) are separated by a certain category boundary, estimates of the location of each target do not interact. Rather, once participants encode a location, information from unselected categories has no effect on stimulus judgment. Consider a trial to the right target in the $20^{\circ}$ separation condition from the previous experiment. (Recall that, in this condition, participants estimated the locations of the $-20^{\circ}, 0^{\circ}$, and $20^{\circ}$ targets.) According to the CA model, when participants saw the $20^{\circ}$ target at the start of this trial, they encoded the fine-grained location of the stimulus and the category to which it belonged, the latter with a great deal of certainty. After the delay, fine-grained information was weighted with the appropriate prototype $\left(90^{\circ}\right)$, and participants moved to the remembered location. Therefore, any information about the $-20^{\circ}$ location that might be held in longer term memory would have no effect on estimation of the $20^{\circ}$ target after encoding.

It is possible, however, that this is not a valid proposal. For instance, if adults' location estimation is affected by the distribu- 
tion of exemplars in the task space-the primary issue we attempted to investigate in this study - then the three target locations in each condition of Experiment 1 might have formed an induced category that spanned the midline category boundary. Consequently, a longer term memory of the $-20^{\circ}$ location built up by repeatedly estimating this location from trial to trial might have affected estimation of the $20^{\circ}$ target (and vice versa). This would have contaminated our measure of geometric prototype effects in Experiment 1.

To explore whether participants' experience with particular locations in one category influenced their memory of targets in the second category, we manipulated how often participants moved to each location in a $20^{\circ}$ separation condition. Two thirds of all trials were to the left target $\left(-20^{\circ}\right)$. The remaining trials were to the center $\left(0^{\circ}\right)$ and right $\left(20^{\circ}\right)$ targets. If a longer term memory of the left target affected participants' responses to the right target in Experiment 1, then there should be an increase in such effects when the memory of the left target is stronger or more accurate. For instance, if estimates of the right target were weakly biased toward the left target in Experiment 1, there should be a stronger leftward bias to the right target when the memory of the left target is strengthened. Alternatively, if remembered information from an unselected spatial category has no effect on estimates of location in a different category - as the CA model predicts - then errors to the right target in the present experiment should be comparable to biases reported in Experiment 1. This would support our conclusion that results from Experiment 1 provided a good estimate of geometric prototype effects at different locations in the left and right categories.

Although we manipulated the frequency with which participants moved to the left, center, and right targets in the present experiment, we kept the total number of trials comparable to Experiment 1 . This required that we reduce the number of delays to two: a short delay (15 s) and a long delay (20 s). Because this might influence prototypical biases, we also included an "unbiased" condition in which participants moved equally often to each location. This condition was very similar to the $20^{\circ}$ separation condition from Experiment 1, allowing us to investigate whether results from Experiment 1 could be replicated.

\section{Method}

\section{Participants}

Eight adults $(M$ age $=22$ years 11.12 months, $S D=42.05$ months $)$ participated in this study. Undergraduate and graduate students were recruited at Indiana University and the University of Iowa, and introductory psychology students were recruited at the University of Iowa. Four participants completed the experiment at Indiana University, whereas the remaining adults participated at the University of Iowa. All participants were right-handed. Approximately equal numbers of women and men participated in each experimental condition.

\section{Apparatus, Materials, Task, and Procedure}

The apparatus, materials, task, and procedure were identical to those of Experiment 1.

\section{Experimental Design}

Participants were randomly assigned to one of two experimental conditions. Participants in both conditions moved to three target locations separated by $20^{\circ}$ : a left, center, and right location. Participants in the no bias condition moved equally often to each target location. Participants in the bias left condition moved to the left target on two thirds of all trials, to the center target on one sixth of all trials, and to the right target on one sixth of all trials. As in the previous experiment, participants completed two experimental sessions. Session 1 began with 15 practice trials, 5 to each target at randomly selected delays. Session 2 began with 6 practice trials, 3 to each target at randomly selected delays. After practice, participants in the no bias condition completed 51 trials in blocks of 15 trials each during each session. There were 7 trials to each target at each of two delays: a short delay $(15 \mathrm{~s})$ and a long delay $(20 \mathrm{~s})$. These delays were chosen because adults made relatively large directional errors at these delays in Experiment 1. Participants in the no bias condition also completed three 0-s delay trials to each target. Participants in the bias left condition completed 52 trials per session in blocks of 15 trials each. There were 15 trials to the biased target and 4 trials to each of the nonbiased targets at each of two delays. In addition, participants completed two 0-s delay trials to each target. All trials were randomized with the constraint that responses to the nonbiased targets never occurred more than twice in succession.

\section{Data Analysis}

The method of analysis was identical to that of Experiment 1. One trial did not meet the initiation time criterion. Overall, $0.54 \%$ of all trials were eliminated after the outlier analyses.

\section{Results}

\section{Constant Directional Error}

Figure 9 shows mean constant directional errors for the left, center, and right targets at each delay in the bias left and no bias conditions. Recall that positive errors for the left and right targets reflect errors away from midline, that is, toward the spatial prototypes. Positive errors for the center target reflect counterclockwise errors. As in Experiment 1, participants in both conditions made outward errors when moving to the left and right targets. Moreover, responses to the center target were quite accurate. Importantly, there were no noticeable differences in performance to the center and right targets across conditions. By contrast, constant errors for the left target were smaller in the bias left condition.

Mean constant directional errors were entered into a three-way ANOVA with bias condition (bias left or no bias) as a betweensubjects variable and delay ( $15 \mathrm{~s}$ or $20 \mathrm{~s}$ ) and target (left, center, or right) as within-subject variables. Results revealed a significant main effect of target, Wilks's $\Lambda=.26, F(2,5)=7.01, p<.05$. As in Experiment 1, errors for the left and right targets were larger than errors for the center target (left: $M=3.13^{\circ}$; center: $M=1.01^{\circ}$; right: $\left.M=2.59^{\circ}\right)$. Results also revealed a significant main effect of delay, Wilks's $\Lambda=.49, F(1,6)=6.27, p<.05$, and a significant Delay $\times$ Bias Condition interaction, Wilks's $\Lambda=.36$, $F(1,6)=10.51, p<.05$. Tests of simple effects conducted at each delay revealed no significant bias condition effects. However, additional simple effects tests revealed a significant delay effect in the no bias condition, $F(1,6)=16.49, p<.01\left(15 \mathrm{~s}: M=2.39^{\circ}\right.$; $\left.20 \mathrm{~s}: M=2.78^{\circ}\right)$, but not in the bias left condition, $F(1,6)=0.26$, $n s\left(15 \mathrm{~s}: M=1.92^{\circ} ; 20 \mathrm{~s}: M=1.87^{\circ}\right)$. As in Experiment 1, errors away from the midline category boundary increased in magnitude over delays in the no bias condition.

\section{Variable Directional Error}

Figure 10 shows mean variable directional errors for each target at each delay in the no bias and bias left conditions. As in 


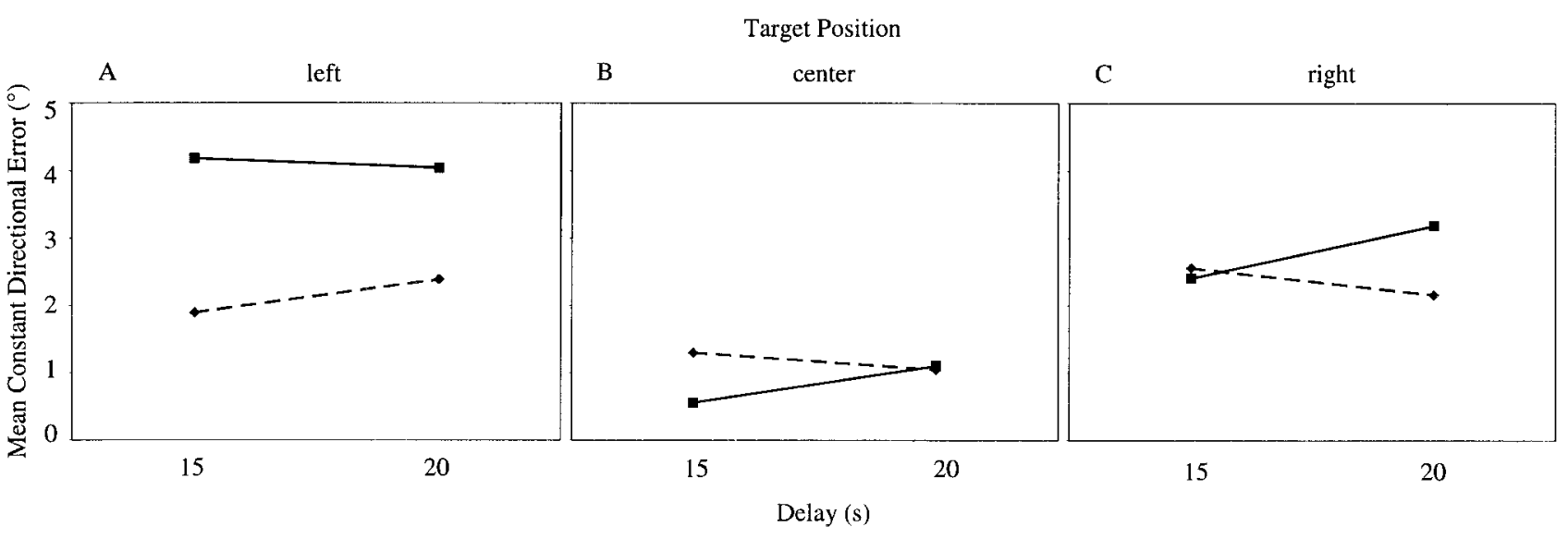

Figure 9. Mean constant directional errors across delays (15 s or $20 \mathrm{~s}$ ) for responses to the left (A), center (B), and right $(\mathrm{C})$ targets in the no bias (solid lines) and bias left (dotted lines) conditions (Experiment 2). Positive errors to the left and right targets reflect errors away from midline, whereas positive errors to the center target reflect counterclockwise errors.

Experiment 1, variable errors for responses to the center target were smaller than for responses to the left and right targets. Furthermore, variability was lower in the no bias condition than in the bias left condition. Mean variable directional errors were entered into a three-way ANOVA with bias condition (bias left or no bias) as a between-subjects variable and delay ( $15 \mathrm{~s}$ or $20 \mathrm{~s}$ ) and target (left, center, or right) as within-subject variables. Results revealed a significant main effect of target, Wilks's $\Lambda=.18, F(2$, $5)=11.23, p<.05$. The target effect reflects the lower variability to the center target, the target aligned with the certain category boundary (left: $M=3.75^{\circ}$; center: $M=2.25^{\circ}$; right: $M=3.23^{\circ}$ ). There were no other significant effects in the overall ANOVA.

\section{Discussion}

The purpose of the present experiment was to determine whether a strong longer term memory of a target in one spatial category (i.e., the left target) would affect the estimation of a target location in an adjacent spatial category (i.e., the right target) when the categories were separated by a certain boundary. As predicted by the CA model, this was not the case; we found no significant differences in responses to the center and right targets across the bias left and no bias conditions. Rather, these data replicated the basic effects from Experiment 1: Participants made outward errors to the right target and were accurate when estimating the location of the center target. Indeed, the magnitudes of these effects were comparable to results from Experiment 1 (see Figures 6 and 9). There was a trend toward a reduction in outward error for the left target in the bias left condition. This probably reflects a type of practice effect. Importantly, however, these bias-related effects did not extend across the midline category boundary. Thus, it appears that the estimates of geometric prototype effects obtained in Experiment 1 were valid; that is, they were not contaminated by cross-category effects. In the next experiment, we used these estimates to test whether participants' memory of spatial locations was affected by the distribution of targets within a spatial category.

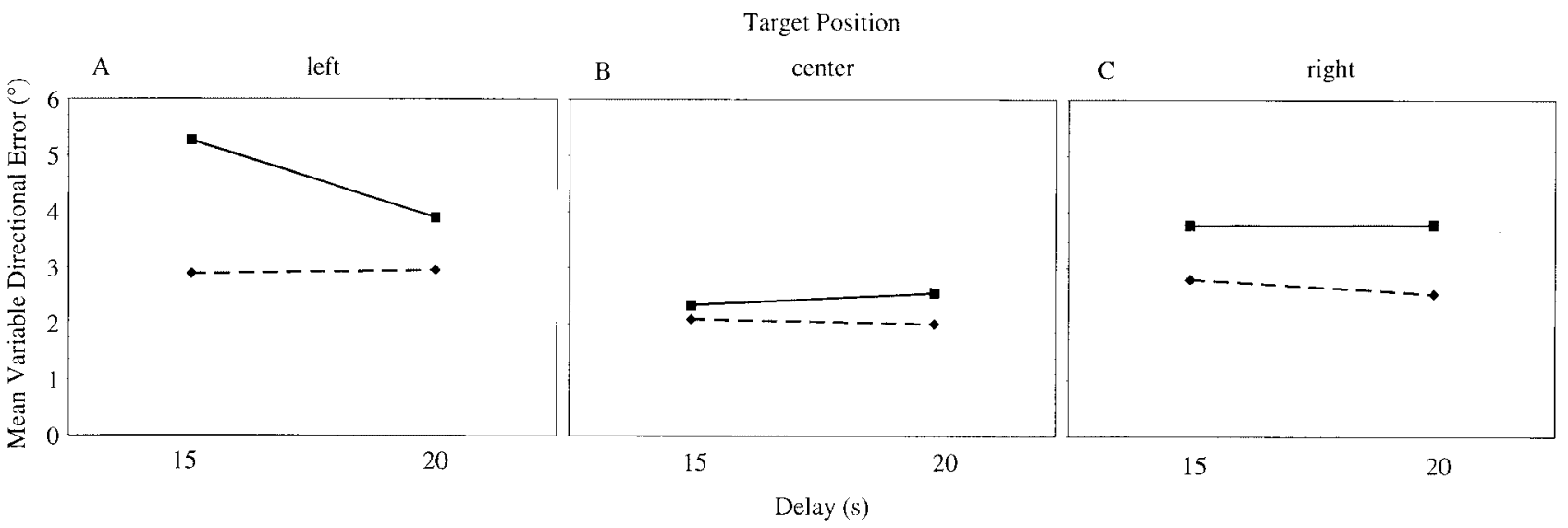

Figure 10. Mean variable directional errors across delays for responses to the left (A), center (B), and right (C) targets in the no bias (solid lines) and bias left (dotted lines) conditions (Experiment 2). 


\section{Experiment 3}

The goal of the present study was to determine whether estimates of location are subject to induced category effects. To accomplish this goal, we examined whether the geometric prototype effects measured in Experiment 1 were modulated by the relative position of exemplars within a spatial category. The design of this experiment is shown in Figure 11. We used locations that were included in Experiment 1: $0^{\circ}, \pm 20^{\circ}, \pm 40^{\circ}, \pm 60^{\circ}$, and $\pm 80^{\circ}$. In each condition, participants estimated the locations of three targets separated by $20^{\circ}$ after 5- to 20-s delays. Across conditions, we varied the layout of the targets in the task space such that all three targets were either within the left category or within the right category. In addition, we varied the rotation of the targets in each category: One subset of targets was close to midline (see Center $20^{\circ}$ in Figure 11), and one subset was far from midline (see Center $60^{\circ}$ in Figure 11).

If participants do not use induced category information, biases at each absolute location should depend solely on target-prototype distance; that is, results for each absolute location should mirror results from Experiment 1. If, however, participants' estimates are affected by induced category information, directional errors across conditions should diverge systematically from the results of Experiment 1 depending on the position of each target within the induced category. For instance, responses to the "outer" targets in each target distribution (i.e., the targets farthest from midline) should be biased inward-toward the center of the induced category-relative to the prototypical biases reported in Experiment 1. By contrast, responses to the "inner" targets in each target distribution (i.e., the targets closest to midline) should be biased out-
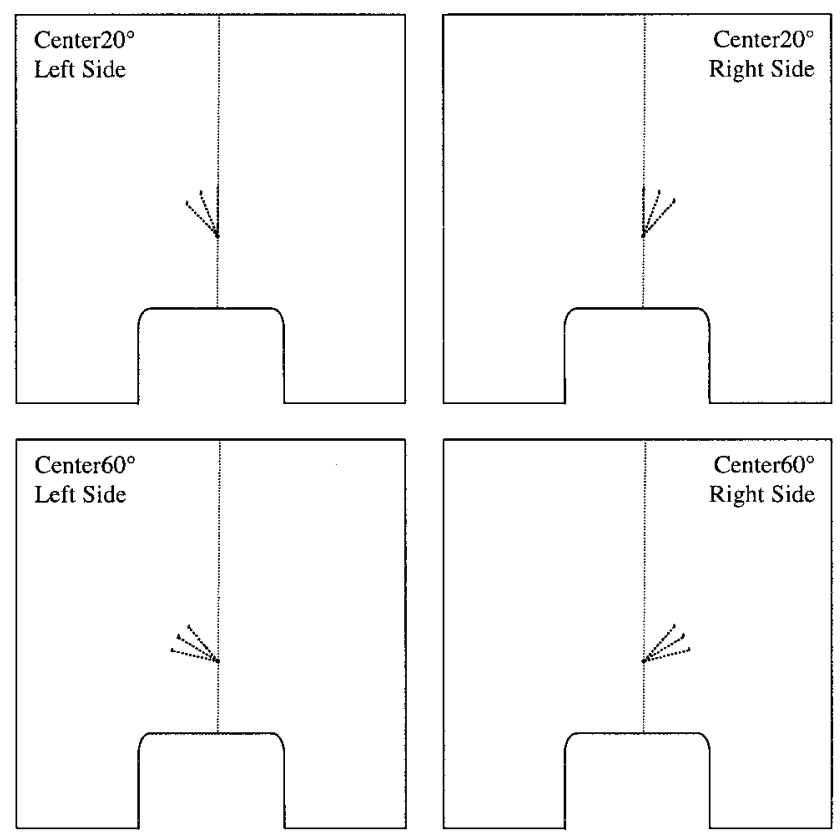

Figure 11. Schematic diagram of target locations presented in each condition of Experiment 3. The dotted line marks the midline axis of the table, which divides the table into left and right categories. Condition names refer to the center target in each distribution (e.g., targets in the Center $20^{\circ}$-right side condition were at $0^{\circ}, 20^{\circ}$, and $40^{\circ}$ ). ward-toward the center of the induced category-relative to the prototypical biases reported in Experiment 1.

\section{Method}

\section{Participants}

Twenty-four adults ( $M$ age $=22$ years 6.0 months, $S D=32.12$ months) participated in this study. Data from 2 additional participants were not included in the final analyses. One adult did not participate in the second data collection session, and the other was excluded because of an experimenter error. Adults were recruited from the general undergraduate and graduate student body at the University of Iowa and from psychology courses at that university. All participants were right-handed. Approximately equal numbers of women and men participated in each condition.

\section{Apparatus, Materials, Task, and Procedure}

The apparatus, materials, task, and procedure were identical to those of Experiments 1 and 2.

\section{Experimental Design}

Participants were randomly assigned to one of four experimental conditions in a full-factorial design. Participants in each condition moved equally often to three target locations separated by $20^{\circ}$ : an inner, center, and outer location (see Figure 11). The target closest to the midline of the table was designated as the inner target, whereas the target furthest from midline was designated as the outer target. We varied the layout of possible target locations across two rotation conditions in either the left or right category. Participants in the center $20^{\circ}$-right side condition saw targets at $0^{\circ}, 20^{\circ}$, and $40^{\circ}$; participants in the center $20^{\circ}$-left side condition saw targets at $0^{\circ},-20^{\circ}$, and $-40^{\circ}$. Similarly, participants in the center $60^{\circ}-$ right side condition saw targets at $40^{\circ}, 60^{\circ}$, and $80^{\circ}$, and participants in the center $60^{\circ}$-left side condition saw targets at $-40^{\circ},-60^{\circ}$, and $-80^{\circ}$. Number of sessions, number of practice trials, and number of trials to each target at each delay (5-20 s) were identical to those of Experiment 1. In addition, we included the same number of 0-s delay trials.

\section{Data Analysis}

The method of analysis was identical to that of Experiments 1 and 2, except that trials were not removed on the basis of the initiation time criterion. As a result of the outlier analysis, an average of $0.51 \%$ of all trials were eliminated.

\section{Results}

\section{Constant Directional Error and Directional Difference Scores}

The goal of the present experiment was to examine whether biases toward geometric prototypes (demonstrated in Experiment 1) are modulated by induced category effects. Participants experienced three target locations, all belonging to one spatial category (e.g., $40^{\circ}, 60^{\circ}$, and $80^{\circ}$ ). We examined whether biases to these locations differed from biases to the same locations in Experiment 1 when there was only one item in each category. Figure 12C and Figure 12D show mean constant directional errors to the inner, center, and outer targets in the center $20^{\circ}$ and center $60^{\circ}$ conditions across delays. Figure 12A and Figure 12B show mean constant directional errors from the analogous targets in Experiment 1. As in previous figures, directional errors were averaged across targets 

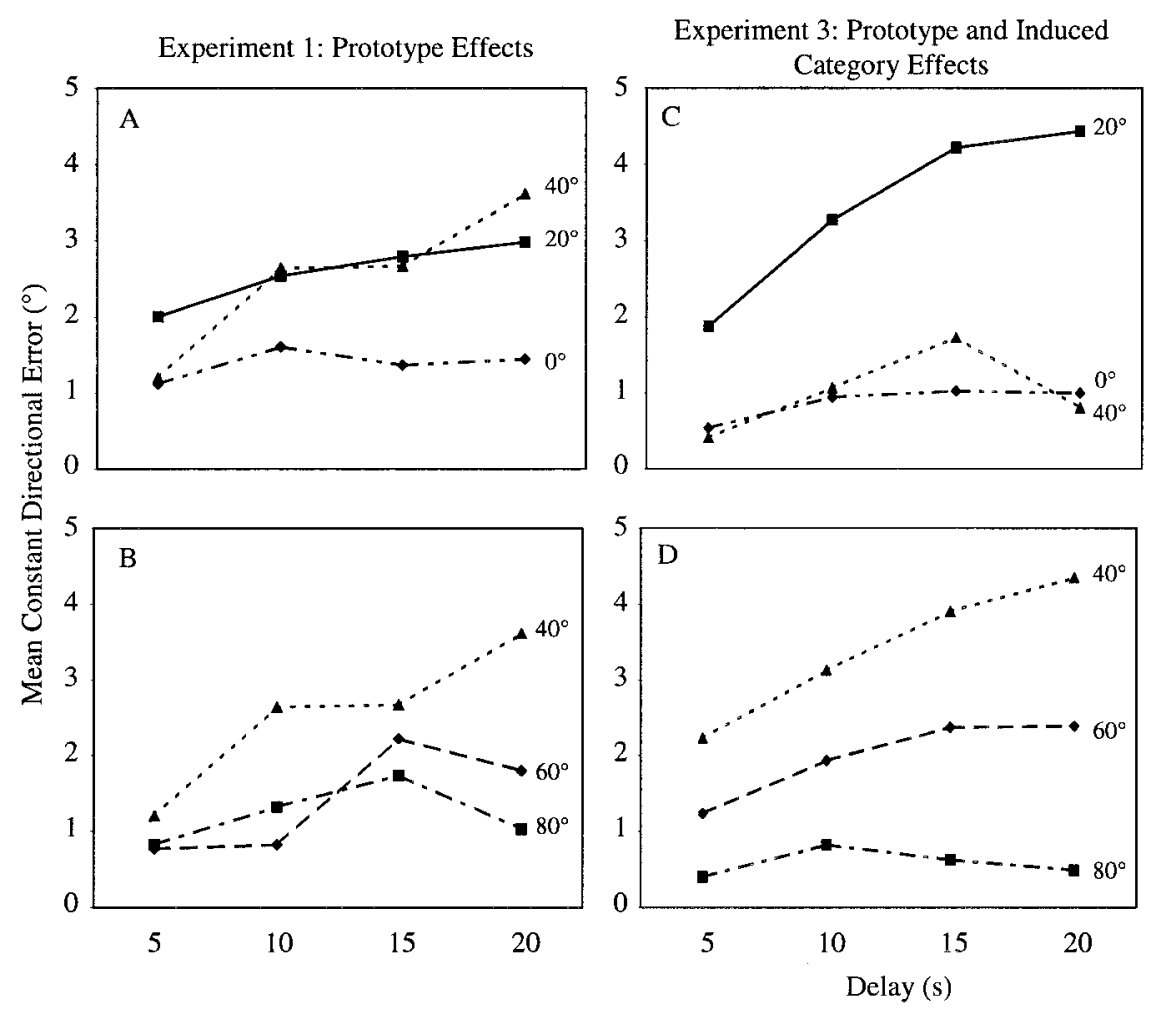

Experiment 3: Induced Category Effects
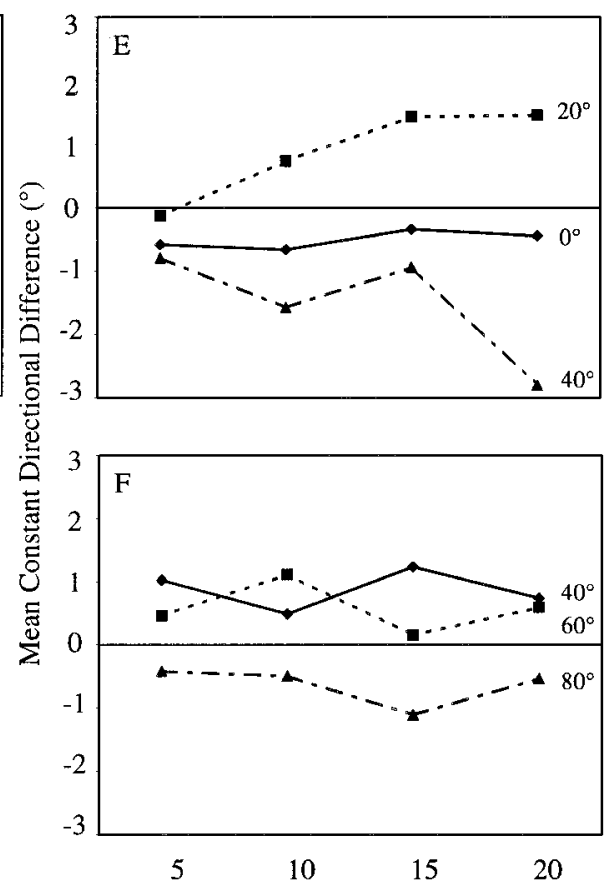

Figure 12. A and B: Mean constant directional errors over delays from Experiment 1 for responses to the target locations included in Experiment 3. Note that data to the $40^{\circ}$ location are duplicated in Panels A and B. Also shown are mean constant directional errors over delays for responses to the inner, center, and outer targets in the center $20^{\circ}$ (C) and center $60^{\circ}$ (D) conditions of Experiment 3, along with mean constant directional difference scores over delays for responses to the inner, center, and outer targets in the center $20^{\circ}(\mathrm{E})$ and center $60^{\circ}(\mathrm{F})$ conditions. Positive difference scores indicate larger biases away from midline relative to the magnitude of the prototype effects at each location (directional difference $=$ mean effect in Experiment $3-$ mean prototype effect in Experiment 1).

in the left and right spatial categories given the symmetry properties of the task space. As can be seen in Figure 12, effects in the present experiment generally replicated findings from Experiments 1 and 2. Specifically, participants made outward directional errors when moving to non $-0^{\circ}$ targets, and these errors increased in magnitude over delays (see Figure 12C and Figure 12D). Furthermore, the magnitude of errors was quite large for targets near midline (i.e., $20^{\circ}$ ) and decreased for targets further from midline (i.e., $80^{\circ}$ ). Finally, responses to the target aligned with the category boundary $\left(0^{\circ}\right)$ were quite accurate across delays.

The central question of interest was whether the layout of targets within a category affected biases away from midline. As can be seen in Figure 12C and Figure 12D, this was indeed the case. For example, biases to the $40^{\circ}$ target in the center $20^{\circ}$ condition (the outer target) were smaller than in Experiment 1 (compare Figure $12 \mathrm{~A}$ and Figure 12C). Similarly, biases to the $80^{\circ}$ target in the center $60^{\circ}$ condition (the outer target) were smaller than in the previous experiment (compare Figure 12B and Figure 12D). To examine these effects in greater detail, we removed effects due to bias toward geometric prototypes from responses obtained in the present study by subtracting the mean constant directional error to each absolute target location from Experiment 1 (i.e., biases in Figure 12C minus biases in Figure 12A and biases in Figure 12D minus biases in Figure 12B). The resulting constant directional difference scores (mean directional error minus mean geometric prototype effect at each location) are shown in Figure 12E and Figure 12F. Positive scores reflect biases away from midline relative to the prototypical biases measured in Experiment 1, whereas negative scores reflect biases toward midline relative to the prototypical biases measured in Experiment 1.

If participants did not use induced category information in the spatial domain, then biases should depend solely on targetprototype distance, and difference scores should be zero. Conversely, if induced categories affect location memory, then difference scores should differ depending on the relative location within the distribution of possible targets. That is, after removal of geometric prototype effects, results should reveal that the inner and outer targets were biased toward the center target in each condition, toward the average of the induced category. As can be seen in Figure 12E and Figure 12F, difference scores in both the center $20^{\circ}$ and center $60^{\circ}$ conditions indicate that participants used induced category information. Difference scores for the outer targets (i.e., $40^{\circ}$ in Figure $12 \mathrm{E}$ and $80^{\circ}$ in Figure $12 \mathrm{~F}$ ) were biased inward or toward the center of the induced category in both conditions, and difference scores for the inner target in the center $60^{\circ}$ condition (i.e., $40^{\circ}$ in Figure $12 \mathrm{~F}$ ) were biased outward or toward the 
center of the induced category. The $40^{\circ}$ data are particularly revealing, because errors for the same absolute spatial location shifted systematically depending on the distribution of the other targets in the task space. Difference scores to the inner and center targets in the center $20^{\circ}$ condition showed a slightly different pattern: Difference scores to the inner target $\left(0^{\circ}\right)$ were near zero error, whereas difference scores to the center target $\left(20^{\circ}\right)$ were positive, reflecting larger biases away from midline relative to prototypical biases.

Mean constant directional difference scores were examined in a four-way ANOVA with rotation (center $20^{\circ}$ or center $60^{\circ}$ ) and side (left or right) as between-subjects variables and delay $(5 \mathrm{~s}, 10 \mathrm{~s}$, $15 \mathrm{~s}$, or $20 \mathrm{~s}$ ) and target (inner, center, or outer) as within-subject variables. Results revealed a significant main effect of target, Wilks's $\Lambda=.60, F(2,19)=6.29, p<.01$, indicating that the induced category effect observed in Figure 12E and Figure 12F was significant. This target effect is illustrated in Figure 13, which depicts mean constant directional difference scores to each target. Difference scores for the inner targets were biased outward (i.e., toward the center of the induced category), whereas difference scores for the outer targets were biased inward (i.e., toward the center of the induced category). One-sample $t$ tests were used to compare directional difference scores for each target and the expected score with no induced category effect (i.e., 0). Difference scores differed significantly from zero error for the outer target, $t(23)=-1.95, p<.05$, but not for the inner and center targets, $t \mathrm{~s}(23)<1.46, n s$.

\section{Variable Directional Error}

Figure 14 shows mean variable directional errors to each target at each delay across the center $20^{\circ}$ and center $60^{\circ}$ conditions. As can be seen, variable errors for the $0^{\circ}$ target (i.e., the inner target in the center $20^{\circ}$ condition) were quite small across all delays. Conversely, variable errors for the non- $0^{\circ}$ targets were larger and increased over delays as memory became more uncertain. These findings generally replicate results from Experiment 1; however, in comparison with data from Experiment 1 (see Figure 7), variability was larger to the $60^{\circ}$ and $80^{\circ}$ targets in the center $60^{\circ}$ condition.

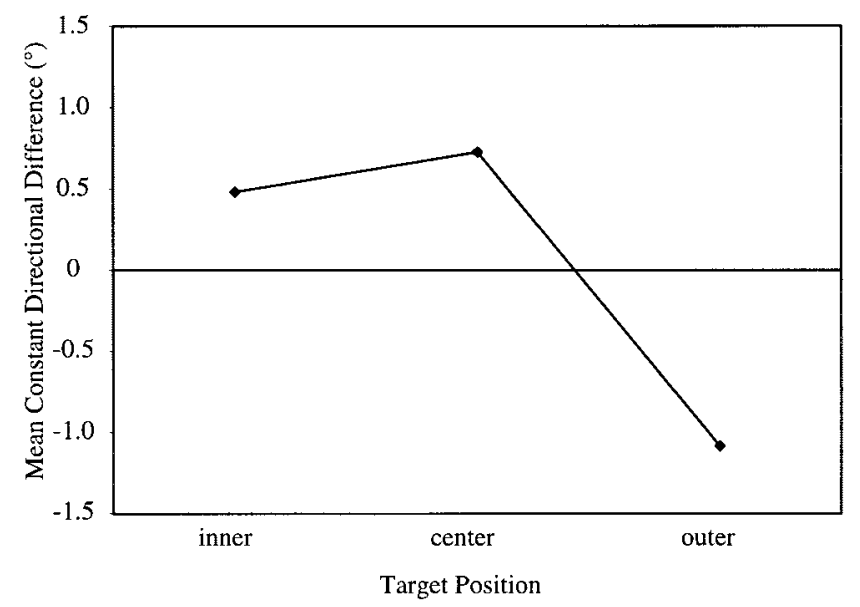

Figure 13. Mean constant directional difference scores to the inner, center, and outer targets (Experiment 3).

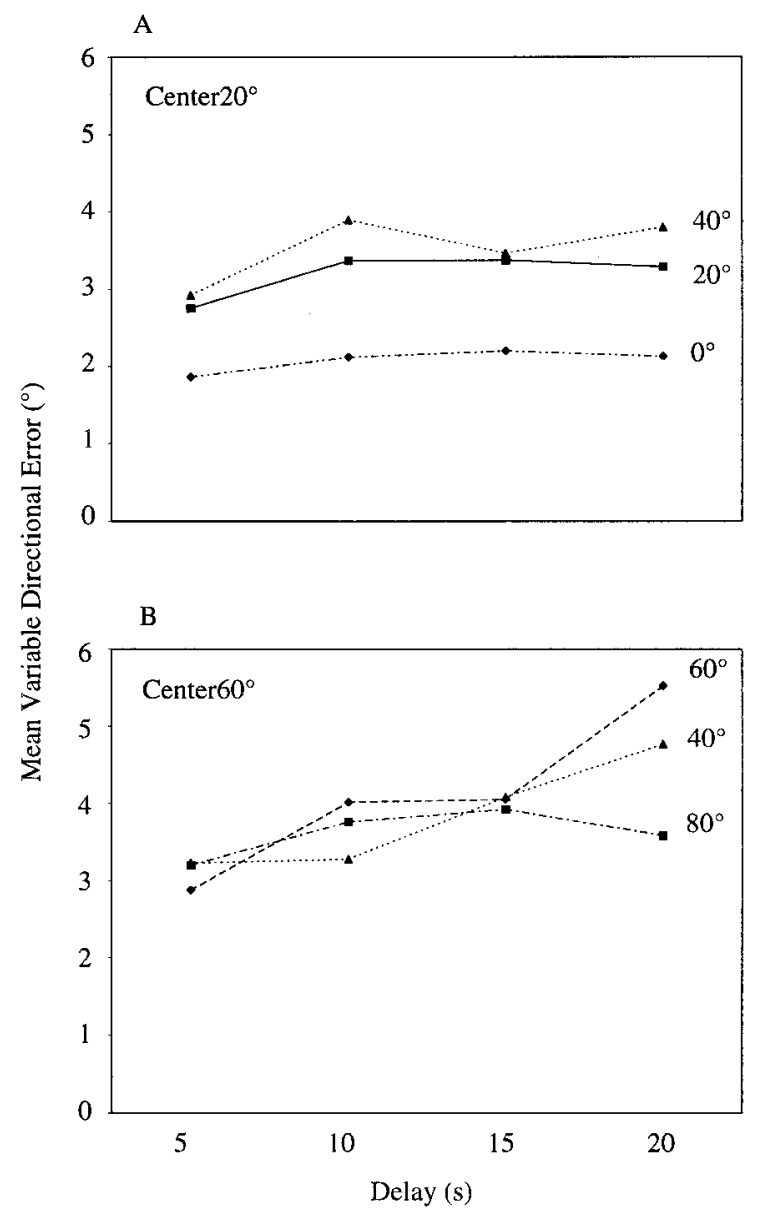

Figure 14. Mean variable directional errors over delays for each target in the center $20^{\circ}(\mathrm{A})$ and center $60^{\circ}$ (B) conditions (Experiment 3).

To investigate these effects in more detail, we examined mean variable directional errors in a four-way ANOVA with rotation (center $20^{\circ}$ or center $60^{\circ}$ ) and side (left or right) as betweensubjects variables and delay $(5 \mathrm{~s}, 10 \mathrm{~s}, 15 \mathrm{~s}$, or $20 \mathrm{~s})$ and target (inner, center, or outer) as within-subject variables. Results revealed significant main effects of delay, Wilks's $\Lambda=.22, F(3$, $18)=21.89, p<.001$, and rotation condition, $F(1,20)=12.61$, $p<.005$. These main effects were subsumed by a significant Delay $\times$ Rotation Condition interaction, Wilks's $\Lambda=.58, F(3$, $18)=4.35, p<.05$. Tests of simple effects revealed that variability increased significantly over delays in the center $60^{\circ}$ condition, $F(3,60)=13.49, p<.01\left(5 \mathrm{~s}: M=3.10^{\circ} ; 10 \mathrm{~s}: M=3.69^{\circ}\right.$; $\left.15 \mathrm{~s}: M=4.02^{\circ} ; 20 \mathrm{~s}: M=4.63^{\circ}\right)$, but not in the center $20^{\circ}$ condition, $F(3,60)=2.65$, $n s\left(5 \mathrm{~s}: M=2.51^{\circ} ; 10 \mathrm{~s}: M=3.13^{\circ}\right.$; $\left.15 \mathrm{~s}: M=3.01^{\circ} ; 20 \mathrm{~s}: M=3.07^{\circ}\right)$. This lack of increase in variability over delays in the center $20^{\circ}$ condition was driven primarily by the low variability to the $0^{\circ}$ target across delays (see Figure 14).

Results also revealed a significant main effect of target, Wilks's $\Lambda=.42, F(2,19)=13.33, p<.001$, and a significant Target $\times$ Rotation Condition interaction, Wilks's $\Lambda=.49, F(2,19)=9.77$, $p<.005$. Tests of simple effects revealed that the difference among targets was significant in the center $20^{\circ}$ condition, $F(2$, 
$40)=14.64, p<.01$ (inner: $M=2.08^{\circ}$; center: $M=3.20^{\circ}$; outer: $M=3.52^{\circ}$ ), but not in the center $60^{\circ}$ condition, $F(2,40)=1.60$, $n s$ (inner: $M=3.84^{\circ}$; center: $M=4.12^{\circ}$; outer: $M=3.62^{\circ}$ ). The difference among targets in the center $20^{\circ}$ condition was largely driven by the low variability to the $0^{\circ}$ target. Moreover, the lack of significant target effects in the center $60^{\circ}$ condition diverged from the results of Experiment 1, in which variability was greater at $40^{\circ}$ than at $60^{\circ}$ and $80^{\circ}$. It is possible that participants' memory of these locations was less certain in the present experiment because there were multiple items in each category.

\section{Discussion}

As in Experiment 1, there were clear geometric prototype effects in the present experiment. Participants' responses to non- $0^{\circ}$ targets were systematically biased away from midline-toward the spatial prototypes-over delays, and directional errors tended to covary with target-prototype distance. Importantly, there were also clear induced category effects. That is, when geometric prototype effects were subtracted from participants' responses, a bias toward the center of the induced category emerged. This effect was most apparent in the center $60^{\circ}$ condition and for the outer target in the center $20^{\circ}$ condition.

Nevertheless, data from the inner and center targets in the center $20^{\circ}$ condition showed a different pattern. Responses to the inner target $\left(0^{\circ}\right)$ were accurate over delays, whereas responses to the center target $\left(20^{\circ}\right)$ were biased more strongly away from midline over delays relative to errors for the same location in Experiment 1 . Although these data differ from results in the center $60^{\circ}$ condition, they may also reflect the use of induced category information. As in Experiments 1 and 2, participants may have treated the $0^{\circ}$ target differently because it was aligned with a certain category boundary. If this were the case, the withincategory distribution of targets in the center $20^{\circ}$ condition may have been composed of only two locations $-20^{\circ}$ and $40^{\circ}$ - shifting the center of the induced category (i.e., the mean) outward. The positive difference scores for the $20^{\circ}$ target may reflect a bias toward the center of this two-item induced category.

Variable errors in the present experiment generally replicated effects from Experiment 1. Consistent with the CA model, there was an increase in variability over delays in the center $60^{\circ}$ condition. In addition, variability was smaller to the $0^{\circ}$ target. There was one notable departure from the effects reported in Experiment 1: Variability to the $60^{\circ}$ and $80^{\circ}$ locations was larger in this experiment than in Experiment 1 . The increase in uncertainty over delays to these locations may reflect a general increase in uncertainty when multiple items are contained within the same category. This effect may have been magnified in the center $60^{\circ}$ condition because the targets were relatively far from the nearest perceptual cue, the midline symmetry axis.

In summary, data from the present experiment are the first, to our knowledge, to show that spatial memory is affected by two types of categorical information: geometric prototypes and the distribution of exemplars within a spatial category. Thus, experience-dependent category processes are not specific to object categorization. Rather, spatial categories can also reflect a group of items organized by common experience.

\section{General Discussion}

The present study explored a fundamental question in the categorization literature: Are there domain-general categorization processes, or are categorization processes specific to each domain? To investigate this question, we examined whether similar categorization processes operate across two dissociable domains, the object and spatial domains. Previous studies have revealed two types of category effects in these domains and suggest that the origin of these effects differs. Research in the spatial domain has shown that adults categorize locations using geometric cues (e.g., Engebretson \& Huttenlocher, 1996; Huttenlocher et al., 1991; Tversky \& Schiano, 1989). In the object domain, data suggest that adults group items using experience-dependent cues (e.g., Huttenlocher et al., 2000; Kruschke, 1996; Nosofsky, 1986). Taken together, these data paint a domain-specific picture of categorization: Categorical biases in each domain originate from different types of information.

We tested this proposal in the present study by investigating whether induced category effects prevalent in the object domain are also seen when adults categorize space. In Experiment 1, we measured the magnitude of spatial prototype effects at individual locations within a left and a right spatial category. As in previous studies by Huttenlocher and colleagues (e.g., Engebretson \& Huttenlocher, 1996; Huttenlocher et al., 1991) and our previous studies with children (Hund \& Spencer, 2001; Schutte \& Spencer, in press; Spencer \& Hund, 2001), adults' estimates of location were biased toward the centers of the left and right categories, and the magnitude of these biases depended on target-prototype distance. Furthermore, consistent with predictions of the CA model, these categorical biases increased as memory became less certain (i.e., more variable) over delays.

Results from Experiments 1 and 2 demonstrated that the left and right categories were separated by a relatively certain boundary. Responses to the center target in both experiments were consistently accurate with low variability. Moreover, strengthening participants' memory of the left target had no significant effects on their memory of a target in the right category. It is important to note that, although we did not find cross-category effects, this may not always be the case. For instance, cross-category effects may arise when adjacent spatial categories are separated by a less certain boundary such as a diagonal symmetry axis (for a discussion of related issues, see Engebretson \& Huttenlocher, 1996; Schiano \& Tversky, 1992; Tversky \& Schiano, 1989).

After measuring geometric prototype effects in the first two experiments, we investigated whether estimates of location were influenced by the distribution of exemplars within a category. In Experiment 3, subsets of targets were positioned at different locations within the left and right categories. When prototype effects were removed, there was a significant bias toward the center of the exemplar distribution to which participants were exposed. For example, difference scores to the $\pm 40^{\circ}$ locations were biased inward in the center $20^{\circ}$ conditions and outward in the center $60^{\circ}$ conditions. Thus, responses to the same absolute locations varied systematically depending on the distribution of targets in the task space, an induced category effect. To our knowledge, these data represent the first demonstration of induced category effects in the spatial domain with adults. Furthermore, they demonstrate for the 
first time that geometric and experience-dependent effects can be separated in studies of spatial categorization.

Although both geometric and experience-dependent information affected adults' estimates of location, our results suggest that geometric information is weighted more heavily in the simple location memory task used here. Specifically, geometric prototype effects measured in Experiment 1 tended to be larger than experience-dependent effects (i.e., difference scores) reported in Experiment 3 . This may reflect differences in the relative certainty of geometric and experience-dependent information in spatial tasks. Geometric cues may be more certain because they are specified by the perceptual structure of the task space (e.g., visible lines and symmetry axes) rather than by information derived from memory. It is possible, however, that adults are able to flexibly weight these two category cues (for related ideas, see Curiel \& Radvansky, 1998; Newcombe \& Huttenlocher, 2000; Schiano \& Tversky, 1992). We suspect, for example, that adults would show larger induced category effects in tasks in which there were clear advantages to using experience-dependent cues. Consistent with this proposal, Curiel and Radvansky (1998) demonstrated that spatial priming effects vary systematically depending on the type of learning task used. In their study, adults learned the locations of 28 objects on a map, but the learning procedure differed across groups. In a naming condition, adults reported the name of an object when cued by a location; in a pointing condition, adults pointed to an object's location when cued by a name. Adults in the naming condition showed strong temporal priming effects in a subsequent testing session. By contrast, adults in the pointing condition showed spatial priming effects.

The induced category effects reported here may also relate to spatial priming in that both effects may stem from associative processes. According to McNamara and colleagues (McNamara, 1986; McNamara \& Diwadkar, 1997), spatial priming results from associations built up in memory as people learn the locations of objects in the world. Locations belonging to the same spatial category and experienced together in time are more strongly associated in memory. As in other associative accounts of memory (e.g., Collins \& Quillian, 1969), retrieval times are faster for strongly associated items. Induced category biases may originate from a similar associative process, that is, from strong associations among within-category items. Participants' experience estimating the same three locations across trials may have created associative links among these items in memory. At recall, participants may have retrieved both the target location and the associated withincategory locations, producing estimates biased toward the center of the target distribution. One clear difference between the present study and studies of spatial priming, however, is that associative links among targets in our task must have been based on the representation of location information. By contrast, strong associations among groups of targets in spatial priming tasks could reflect object-object associations rather than location-location associations.

In addition to these links with spatial priming, our data provide a bridge between how young children and adults form spatial categories. Data from several recent studies show that young children's estimates of location are strongly biased toward induced category information (Schutte \& Spencer, in press; Spencer et al., 2001). For example, Spencer et al. (2001) reported that 2-yearolds' memory responses were biased toward an average target location in a sandbox task. Across five "training" trials, these researchers hid toys at randomly selected locations that varied across a 20.3-cm (8-in.) spatial range. On the sixth trial, a toy was hidden at a "test" location $20.3 \mathrm{~cm}$ from the average training location. Two-year-olds' responses were biased toward the average training location on the test trial. Moreover, these induced category biases were stronger than geometric prototype effects. This difference in weighting relative to the present findings suggests that the relative weighting of these two types of category cues may change dramatically over development (see also Newcombe \& Huttenlocher, 2000).

\section{The CA Model: Toward a Domain-General Account of Categorization}

Data from the present study, in conjunction with results from the object categorization literature, demonstrate that the use of experience-dependent information is domain general. Nevertheless, these commonalities alone do not indicate that a domaingeneral process underlies category formation. It is possible, for instance, that similarities across domains reflect similarities in the types of information used in each domain (a memory of items experienced in a task). Careful inspection, however, might reveal domain-specific characteristics of how this information is represented in each domain or unique signatures of the processes that operate on this information in each domain. Thus, although the data presented here create links across dissociable domains, this is only a first step. Systematic testing and comparison of formal, process-based models of categorization in each domain are needed.

The CA model is the only formal model that has been tested in both the spatial and object categorization domains. This model clearly has several strengths. Central to the present study, the model can account for both geometric prototype effects in the spatial domain (see Huttenlocher et al., 1991) and induced category effects in the object domain (see Huttenlocher et al., 2000). Furthermore, the model can account for several empirical results from the present study, including the increase in geometric prototype effects over delays and the associated increase in variable errors in Experiment 1.

Nevertheless, there are several key limitations of the CA model. First, both geometric prototype and induced category effects have been mathematically implemented in the same way in the model. Thus, one cannot isolate effects that might be unique to each type of categorization process. For instance, if prototype effects are explicitly linked to the geometry of the task space, one might expect prototypical biases to be relatively constant during a data collection session. By contrast, induced category effects should change during a session as participants gain experience estimating the target locations.

A second limitation is related to the first: The CA model does not formally specify the origin of geometric prototype and induced category effects, that is, the processes that produce these two types of effects. Our data suggest that there may be important differences in this regard. Spatial prototype effects appear to be strongly linked to perceptual processes, processes that pick up information about visible reference lines and extract axes of symmetry (see, e.g., Beh, Wenderoth, \& Purcell, 1971; Li \& Westheimer, 1997; Wenderoth, 1997). By contrast, induced category effects are linked to memorial processes. Formalizing these processes would allow for direct 
comparisons with models of object-semantic categorization that construct prototype-like effects over learning on the basis of the characteristics of exemplars (e.g., Kruschke, 1996). A final limitation also reflects a need for greater specification of process in the model: The CA model has no mechanism for producing timedependent effects. To simulate the delay-dependent results from Experiment 1, one would need to modify the certainty of finegrained information continuously "by hand" to produce a more biased outcome. Such an ad hoc solution is underconstrained and fails to predict a priori the details of the time-dependent effects reported here. Therefore, although the CA model captures many of the qualitative results reported in this article, as well as data from the extant literature, we contend that the model needs further development before it can fully explain the domain-general nature of categorization behaviors in both the object and spatial domains.

\section{Are There "Geometric" Effects in the Object Domain?}

The present study focused on the spatial domain in an effort to link categorization phenomena across the "what" and "where" systems. Although our induced category results accomplished this objective, our results also point toward a possible domain-specific aspect of spatial categorization. In addition to induced category effects, we found evidence of geometric category effects (see also Engebretson \& Huttenlocher, 1996; Huttenlocher et al., 1994; Sandberg et al., 1996). According to the CA model, category boundaries in the spatial domain are represented explicitly (Huttenlocher et al., 1991). Moreover, boundaries are linked to the perceptual structure of the task space; people use visible frames and axes of symmetry to organize space into categories. Several researchers have suggested that people may be able to "mentally impose" spatial category boundaries based on task-specific experience (Huttenlocher et al., 1991). That is, people might flexibly use category boundaries to optimize performance given particular task demands. For example, Tversky and Schiano (Schiano \& Tversky, 1992; Tversky \& Schiano, 1989) reported that adults used different category boundaries when figures were presented as "graphs" than when they were presented as "maps." It is important to note, however, that even when adults flexibly use category boundaries, these boundaries are still linked to the perceptual structure of the task space (i.e., reference lines and symmetry axes). Thus, explicitly represented category boundaries linked to perceptual cues have a major influence on how people categorize space.

Do similar geometric effects operate in the object categorization domain? Several researchers have proposed that category boundaries are represented explicitly. For instance, Ashby and colleagues (Ashby \& Gott, 1988; Ashby \& Maddox, 1993; Maddox \& Ashby, 1996) proposed that decision rules, or boundaries, organize a stimulus space into regions corresponding to differing responses. When people are presented with a stimulus (e.g., a shape that might vary in size and color), they decide which region it belongs to and produce the response associated with that region.

Generally, boundaries affecting object categorization are more abstract than the spatial boundaries just described. Nevertheless, decision rules or boundaries are linked to values along particular stimulus dimensions regardless of complexity. For example, according to Ashby and Gott (1988), people first adopt simple decision rules. People might decide that all shapes bigger than $X$ units belong to category $A$, whereas those smaller than $X$ belong to $B$. Simple rules such as this one are used as long as the resulting category decisions are acceptable (i.e., accurate enough given the task demands). However, people might formulate more complex rules when simple rules become unacceptable. That is, they might decide that shapes bigger than $X$ units and red belong to category $A$, whereas all others belong to category $B$. As this example illustrates, decision rules or boundaries can be quite complex. Nevertheless, they are still linked to perceivable stimulus values. In this sense, one could claim that decision rules are linked to the structure of the stimulus space, a multidimensional space defined by the stimulus dimensions, much like spatial boundaries are linked to the stimulus space, the geometric dimensions of a task space.

Although this proposal offers parallels between spatial and object categorization, other researchers have proposed that object categorization depends solely on experience with exemplars and not on explicitly represented boundaries. For instance, Nosofsky (1986) proposed that people represent exemplars as points in a multidimensional space with dimensions that correspond to the dimensions along which stimuli vary. When asked to categorize a novel exemplar, people compare this stimulus with the set of remembered exemplars. The resultant category response depends on the similarity between the novel and the stored exemplars. Importantly, category boundaries are not represented explicitly. Rather, boundary-like effects emerge between clusters of exemplars that belong to different categories.

As these alternative proposals illustrate, boundaries might influence object categorization in numerous ways. Thus, on the basis of current theoretical and empirical work, it is not clear whether there is an analog to geometric effects in the object domain, nor is it clear whether geometric and experience-dependent effects can be separated in the object domain as we have done here in the spatial domain. Nevertheless, our data demonstrate that there are exciting parallels in categorization phenomena across domains.

\section{References}

Ashby, F. G., \& Gott, R. E. (1988). Decision rules in the perception and categorization of multidimensional stimuli. Journal of Experimental Psychology: Learning, Memory, and Cognition, 14, 33-53.

Ashby, F. G., \& Maddox, W. T. (1993). Relations between prototype, exemplar, and decision bound models of categorization. Journal of Mathematical Psychology, 37, 372-400.

Barsalou, L. W. (1983). Ad hoc categories. Memory \& Cognition, 11, 211-227.

Beh, H., Wenderoth, P., \& Purcell, A. (1971). The angular function of a rod-and-frame illusion. Perception \& Psychophysics, 9, 353-355.

Carnahan, H. (1998). Manual asymmetries in responses to rapid target movement. Brain \& Cognition, 37, 237-253.

Cecala, A. J., \& Garner, W. R. (1986). Internal frame of reference as a determinant of the oblique effect. Journal of Experimental Psychology: Human Perception and Performance, 12, 314-323.

Chieffi, S., \& Allport, D. A. (1997). Independent coding of target distance and direction in visuo-spatial working memory. Psychological Research, 60, 244-250.

Chieffi, S., Allport, D. A., \& Woodin, M. (1999). Hand-centered coding of target location in visuo-spatial working memory. Neuropsychologia, 37, 495-502.

Clayton, K., \& Habibi, A. (1991). Contribution of temporal contiguity to 
the spatial priming effect. Journal of Experimental Psychology: Learning, Memory, and Cognition, 17, 263-271.

Collins, A. M., \& Quillian, M. R. (1969). Retrieval time from semantic memory. Journal of Verbal Learning and Verbal Behavior, 8, 240-247.

Curiel, J. M., \& Radvansky, G. A. (1998). Mental organization of maps. Journal of Experimental Psychology: Learning, Memory, and Cognition, 24, 202-214.

di Pellegrino, G., \& Wise, S. P. (1993). Visuospatial versus visuomotor activity in the premotor and prefrontal cortex of a primate. Journal of Neuroscience, 13, 1227-1243.

Elliott, D., \& Madalena, J. (1987). The influence of premovement visual information on manual aiming. Quarterly Journal of Experimental Psychology, 39A, 541-559.

Engebretson, P. H., \& Huttenlocher, J. (1996). Bias in spatial location due to categorization: Comment on Tversky and Schiano. Journal of Experimental Psychology: General, 125, 96-108.

Fisk, J. D., \& Goodale, M. A. (1985). The organization of eye and limb movements during unrestricted reaching to targets in contralateral and ipsilateral visual space. Experimental Brain Research, 60, 159-178.

Haggard, P., Newman, C., Blundell, J., \& Andrew, H. (2000). The perceived position of the hand in space. Perception \& Psychophysics, 68, 363-377.

Humphreys, G. W., \& Riddoch, J. (1987). To see but not to see: A case study of visual agnosia. Hove, England: Erlbaum.

Hund, A. M., \& Spencer, J. P. (2001). Developmental changes in the relative weighting of geometric and experience-dependent location cues. Manuscript submitted for publication.

Huttenlocher, J., Hedges, L. V., \& Duncan, S. (1991). Categories and particulars: Prototype effects in estimating spatial location. Psychological Review, 98, 352-376.

Huttenlocher, J., Hedges, L., \& Prohaska, V. (1988). Hierarchical organization in ordered domains: Estimating the dates of events. Psychological Review, 95, 471-484.

Huttenlocher, J., Hedges, L. V., \& Vevea, J. L. (2000). Why do categories affect stimulus judgment? Journal of Experimental Psychology: General, 129, 220-241.

Huttenlocher, J., Newcombe, N., \& Sandberg, E. H. (1994). The coding of spatial location in young children. Cognitive Psychology, 27, 115-147.

Kay, P., \& McDaniel, C. K. (1978). The linguistic significance of the meanings of basic color terms. Language, 54, 610-646.

Koehler, J. J. (1996). The base rate fallacy reconsidered: Descriptive, normative, and methodological challenges. Behavioral and Brain Sciences, 19, 1-17.

Kruschke, J. K. (1996). Base rates in category learning. Journal of Experimental Psychology: Learning, Memory, and Cognition, 22, 3-26.

Laeng, B., Peters, M., \& McCabe, B. (1998). Memory for locations within regions: Spatial biases and visual hemifield differences. Memory \& Cognition, 26, 97-107.

Levine, D. N., Warach, J., \& Farah, M. J. (1985). Two visual systems in mental imagery: Dissociation of "what" and "where" in imagery disorders due to bilateral posterior cerebral lesions. Neurology, 35, 10101018.

Li, W., \& Westheimer, G. (1997). Human discrimination of the implicit orientation of simple symmetrical patterns. Vision Research, 37, 565572.

Maddox, W. T., \& Ashby, F. G. (1996). Perceptual separability, decisional separability, and the identification-speeded classification relationship. Journal of Experimental Psychology: Human Perception and Performance, 22, 795-817.

McIntyre, J., Stratta, F., \& Lacquaniti, F. (1997). Viewer-centered frame of reference for pointing to memorized targets in three-dimensional space. Journal of Neurophysiology, 78, 1601-1618.

McIntyre, J., Stratta, F., \& Lacquaniti, F. (1998). Short-term memory for reaching to visual targets: Psychophysical evidence for body-centered reference frames. Journal of Neuroscience, 18, 8423-8435.

McNamara, T. P. (1986). Mental representations of spatial relations. Cognitive Psychology, 18, 87-121.

McNamara, T. P., \& Diwadkar, V. A. (1997). Symmetry and asymmetry of human spatial memory. Cognitive Psychology, 34, 160-190.

McNamara, T. P., Halpin, J. A., \& Hardy, J. K. (1992). Spatial and temporal contributions to the structure of spatial memory. Journal of Experimental Psychology: Learning, Memory, and Cognition, 18, 555564.

Miller, E. K., \& Desimone, R. (1994). Parallel neuronal mechanisms for short-term memory. Science, 263, 520-522.

Miller, E. K., Erickson, C. A., \& Desimone, R. (1996). Neural mechanisms of visual working memory in prefrontal cortex of the macaque. Journal of Neuroscience, 16, 5154-5167.

Miller, E. K., Li, L., \& Desimone, R. (1991). A neural mechanism for working and recognition memory in inferior temporal cortex. Science, 254, 1377-1379.

Nelson, T. O., \& Chaiklin, S. (1980). Immediate memory for spatial location. Journal of Experimental Psychology: Human Learning and Memory, 6, 529-545.

Newcombe, N., \& Huttenlocher, J. (2000). Making space: The development of spatial representation and reasoning. Cambridge, MA: MIT Press.

Newcombe, N., Huttenlocher, J., Sandberg, E., Lie, E., \& Johnson, S. (1999). What do misestimations and asymmetries in spatial judgment indicate about spatial representation? Journal of Experimental Psychology: Learning, Memory, and Cognition, 25, 986-996.

Nosofsky, R. M. (1986). Attention, similarity, and the identificationcategorization relationship. Journal of Experimental Psychology: General, 115, 39-57.

Posner, M. I., \& Keele, S. W. (1968). On the genesis of abstract ideas. Journal of Experimental Psychology, 77, 353-363.

Rao, S. C., Rainer, G., \& Miller, E. K. (1997). Integration of what and where in the primate prefrontal cortex. Science, 276, 821-824.

Rosch, E. (1973). On the internal structure of perceptual and semantic categories. In T. E. Moore (Ed.), Cognitive development and the acquisition of language (pp. 111-144). San Diego, CA: Academic Press.

Rosch, E. (1975). Cognitive reference points. Cognitive Psychology, 7, 532-547.

Rosch, E., Simpson, C., \& Miller, R. S. (1976). Structural bases of typicality effects. Journal of Experimental Psychology: Human Perception and Performance, 2, 491-502.

Rothbart, M., Davis-Stitt, C., \& Hill, J. (1997). Effects of arbitrarily placed category boundaries on similarity judgments. Journal of Experimental Social Psychology, 33, 122-145.

Sandberg, E. H., Huttenlocher, J., \& Newcombe, N. (1996). The development of hierarchical representation of two-dimensional space. Child Development, 67, 721-739.

Schiano, D. J., \& Tversky, B. (1992). Structure and strategy in encoding simplified graphs. Memory \& Cognition, 20, 12-20.

Schutte, A. R., \& Spencer, J. P. (in press). Generalizing the dynamic field theory of the A-not-B error beyond infancy: Three-year-olds' delay- and experience-dependent location memory biases. Child Development.

Shoqierat, M. A., \& Mayes, A. R. (1991). Disproportionate incidental spatial-memory and recall deficits in amnesia. Neuropsychologia, 29, 749-769.

Spencer, J. P., \& Hund, A. M. (2001). Are we there yet? Spatial memory models fail to capture both geometric and experience-dependent biases in children's memory for locations. Manuscript submitted for publication.

Spencer, J. P., Smith, L. B., \& Thelen, E. (2001). Tests of a dynamic systems account of the A-not-B error: The influence of prior experience 
on the spatial memory abilities of 2-year-olds. Child Development, 72, 1327-1346.

Suzuki, W. A., Miller, E. K., \& Desimone, R. (1997). Object and place memory in the macaque entorhinal cortex. Journal of Neurophysiology, 78, 1062-1081.

Tversky, B., \& Schiano, D. J. (1989). Perceptual and conceptual factors in distortions in memory for graphs and maps. Journal of Experimental Psychology: General, 118, 387-398.

Ungerleider, L. G., \& Mishkin, M. (1982). Two cortical visual systems. In D. J. Ingle, M. A. Goodale, \& R. J. W. Mansfield (Eds.), Analysis of visual behavior (pp. 549-586). Cambridge, MA: MIT Press.

Wenderoth, P. (1997). The role of implicit axes of bilateral symmetry in orientation processing. Australian Journal of Psychology, 49, 176-181.
Wenderoth, P., Johnstone, S., \& van der Zwan, J. (1989). Two-dimensional tilt illusions induced by orthogonal plaid patterns: Effects of plaid motion, orientation, spatial separation, and spatial frequency. Perception, 18, 25-38.

Wilson, F. A. W., Scalaidhe, S. P., \& Goldman-Rakic, P. S. (1993). Dissociation of object and spatial processing domains in primate prefrontal cortex. Science, 260, 1955-1958.

Winter, D. A. (1990). Biomechanics and motor control of human movement (2nd ed.). New York: Wiley.

Received January 2, 2001

Revision received June 8, 2001

Accepted August 1, 2001

\section{Members of Underrepresented Groups: Reviewers for Journal Manuscripts Wanted}

If you are interested in reviewing manuscripts for APA journals, the APA Publications and Communications Board would like to invite your participation. Manuscript reviewers are vital to the publications process. As a reviewer, you would gain valuable experience in publishing. The $\mathrm{P} \& \mathrm{C}$ Board is particularly interested in encouraging members of underrepresented groups to participate more in this process.

If you are interested in reviewing manuscripts, please write to Demarie Jackson at the address below. Please note the following important points:

- To be selected as a reviewer, you must have published articles in peer-reviewed journals. The experience of publishing provides a reviewer with the basis for preparing a thorough, objective review.

- To be selected, it is critical to be a regular reader of the five to six empirical journals that are most central to the area or journal for which you would like to review. Current knowledge of recently published research provides a reviewer with the knowledge base to evaluate a new submission within the context of existing research.

- To select the appropriate reviewers for each manuscript, the editor needs detailed information. Please include with your letter your vita. In your letter, please identify which APA journal(s) you are interested in, and describe your area of expertise. Be as specific as possible. For example, "social psychology" is not sufficient-you would need to specify "social cognition" or "attitude change" as well.

- Reviewing a manuscript takes time (1-4 hours per manuscript reviewed). If you are selected to review a manuscript, be prepared to invest the necessary time to evaluate the manuscript thoroughly.

Write to Demarie Jackson, Journals Office, American Psychological Association, 750 First Street, NE, Washington, DC 20002-4242. 